\title{
Localization for One Dimensional Long Range Random Hamiltonians*
}

\author{
Vojkan Jakšić ${ }^{1}$ and Stanislav Molchanov ${ }^{2}$ \\ ${ }^{1}$ Department of Mathematics and Statistics \\ University of Ottawa, 585 King Edward Avenue \\ Ottawa, ON, K1N 6N5, Canada \\ ${ }^{2}$ Department of Mathematics \\ University of North Carolina \\ Charlotte, NC 28223, USA
}

April 28, 1998

\begin{abstract}
We study spectral properties of random Schrödinger operators $h_{\omega}=h_{0}+v_{\omega}(n)$ on $l^{2}(\mathbf{Z})$ whose free part $h_{0}$ is long range. We prove that the spectrum of $h_{\omega}$ is pure point for typical $\omega$ whenever the off-diagonal terms of $h_{0}$ decay as $|i-j|^{-\gamma}$ for some $\gamma>8$.
\end{abstract}

${ }^{*}$ To appear in Reviews in Mathematical Physics 


\section{Introduction}

In this paper we study spectral properties of random Schrödinger operators

$$
h_{\omega}=h_{0}+v_{\omega}(n)
$$

on $l^{2}(\mathbf{Z})$ where $h_{0}$ is not the usual free Hamiltonian but only a bounded self-adjoint operator with some off-diagonal decay. We assume that $v_{\omega}(n)$ are independent and identically distributed random variables on a probability space $(\Omega, \mathcal{F}, P)$ with density $p(x)$. We denote by $\mathcal{V}$ the support of the probability measure $p(x) d x$. In the sequel, unless otherwise stated, we will always assume that $\mathcal{V}$ is compact. We further assume that $h_{0}$ is translation invariant, namely that there is a function $j: \mathbf{Z} \mapsto \mathbf{C}$ such that

$$
\left(h_{0} \psi\right)(n)=\sum_{m} j(n-m) \psi(m) .
$$

We are interested in the case where

$$
|j(n)| \leq C\langle n\rangle^{-\gamma}
$$

for some constants $\gamma>1$ and $C>0$. Here and in the sequel, $\langle x\rangle=\left(1+x^{2}\right)^{1 / 2}$. Let

$$
\hat{j}(\varphi)=\sum_{n} j(n) e^{i n \varphi}, \quad \varphi \in[-\pi, \pi] .
$$

If $(1.3)$ holds, then $\sigma\left(h_{0}\right)=[\min \hat{j}(\varphi), \max \hat{j}(\varphi)]$. We remark that if in addition the function $\hat{j}(\varphi)$ is piecewise monotone, then the spectrum of $h_{0}$ is purely absolutely continuous.

Let $\Sigma=\sigma\left(h_{0}\right)+\mathcal{V}$. The standard argument yields that $\sigma\left(h_{\omega}\right)=\Sigma P$-a.s. Furthermore, there exist sets $\Sigma_{a c}, \Sigma_{s c}, \Sigma_{p p} \subset \mathbf{R}$ such that $P$-a.s., $\sigma_{a c}\left(h_{\omega}\right)=\Sigma_{a c}, \sigma_{s c}\left(h_{\omega}\right)=\Sigma_{s c}, \sigma_{p p}\left(h_{\omega}\right)=\Sigma_{p p}$, and $\Sigma=\Sigma_{a c} \cup \Sigma_{s c} \cup \Sigma_{p p}$, see e.g. [CFKS]. As usual, we denote $\Sigma_{c}=\Sigma_{a c} \cup \Sigma_{s c}$. We are interested under what conditions the spectrum of $h_{\omega}$ is pure point $P$-a.s., or in other words, under what conditions is $\Sigma_{c}=\emptyset$. To the best of our knowledge, the only known result is proven in [AM]: If $h_{\omega}=h_{0}+\lambda v_{\omega}(n)$, then for $|\lambda|$ sufficiently large $\Sigma_{c}=\emptyset$. This result also holds for the $d$-dimensional analog of (1.1) if $\gamma>d$. Simon and Spencer [SS] have studied deterministic Hamiltonians of the form (1.1), and they derived a set of sufficient conditions under which these operators have no absolutely continuous spectrum. Their results motivated our work, and we will discuss them below.

Since the model (1.1) has been rarely studied, we will briefly discuss on the typical example some of its main features. Assume that $j(0)=-1, j(n)=|n|^{-\gamma} / 2 \zeta(\gamma)$, where $\zeta$ is the usual Riemann zeta function. Then the long range Laplacian $h_{0}$ generates a random walk on $\mathbf{Z}$ which is transient if $\gamma<2$, and recurent if $\gamma \geq 2$. Let $h(\varphi)=-\hat{j}(\varphi)$. The function $h$ is strictly 
monotone and differentiable on $(0, \pi)$, and we denote its inverse by $h^{-1}(E), E \in \sigma\left(-h_{0}\right)$. Note that $\sigma\left(-h_{0}\right)=[0, h(\pi)]$. The density of states of $-h_{0}$, constructed using the periodic boundary conditions, is $n(E)=h^{-1}(E) / \pi$. The asymptotics of $n(E)$ as $E \downarrow 0$ is computed from the asymptotics of $h(\varphi)$ as $\varphi \downarrow 0$. It is not difficult to show that as $E \downarrow 0$,

$$
n(E) \sim \begin{cases}c_{\gamma} E^{1 / 2} & \text { if } \gamma>3, \\ c_{\gamma} E^{1 /(\gamma-1)} & \text { if } 1<\gamma<3,\end{cases}
$$

where $c_{\gamma}$ 's are computable constants. One can also compute the asymptotics of $n(E)$ if $\gamma=3$, which includes logarithmic terms. Thus, if $\gamma=1+2 / d$, the operator $h_{0}$ has some characteristic features of the usual free Laplacian on $\mathbf{Z}^{d}$ defined by

$$
\left(\Delta_{d} \psi\right)(\mathbf{n})=\frac{1}{2 d} \sum_{\mathbf{m},|\mathbf{m}-\mathbf{n}|_{+}=1}(\psi(\mathbf{m})-\psi(\mathbf{n})),
$$

where $|\mathbf{n}|_{+}=\sum\left|n_{i}\right|$. We remaind the reader that the random walk generated by $\Delta_{d}$ is transient if $d>2$ and recurent if $d=1,2$. Furthermore, $\sigma\left(-\Delta_{d}\right)=[0,2]$, and its density of states, $n_{d}(E)$, satisfies $n_{d}(E) \sim c_{d} E^{d / 2}$ as $E \downarrow 0$. These observations suggest that it is possible that in the weak coupling regime and for $\gamma$ sufficiently close to 1 the model (1.1) has delocalized states. On the other hand, it is natural to conjecture that mathematical localization holds whenever $\gamma>2$. This paper deals with this conjecture. In particular, we will show that $\Sigma_{c}=\emptyset$ under the following conditions:

a) $\gamma>8$.

b) $\hat{j}(\varphi)$ is an even real function strictly monotone on $[0, \pi]$, or, $\operatorname{ess}_{\operatorname{supp}}\left|v_{\omega}(n)\right|$ is sufficiently large.

If $\gamma>4$ and b) holds, we will show using the theorem of Simon and Spencer [SS] that $\Sigma_{a c}=\emptyset$.

Let us state our results precisely. We recall that $\mathcal{V}$ is the support of the measure $p(x) d x$.

Theorem 1.1 Assume that $\hat{j}(\varphi)$ is an even real function strictly monotone on $[0, \pi]$, and that $\operatorname{int}(\mathcal{V}) \neq \emptyset$.

1. If $\gamma>4$ then $\Sigma_{a c}=\emptyset$.

2. If $\gamma>8$ then $\Sigma_{c}=\emptyset$.

Remark 1. The first condition of the theorem is satisfied, for example, if $j(n)$ is an even positive sequence such that $n j(n)$ is convex for $n>0$ (see Theorem $4.1 \mathrm{in}[\mathrm{K}]$ ). In particular, the theorem holds if $j(n)=|n|^{-\gamma}$. The second condition of the theorem, int $\mathcal{V} \neq \emptyset$, is a condition on the density $p(x)$. It is satisfied, for example, if $p$ is non-zero and continuous on some interval.

Remark 2. Our estimates give some control of the decay of the eigenfunctions of $h_{\omega}$. For example, if $j(n)$ decays faster then any polynomial (i.e. $\hat{j}(\phi)$ is $C^{\infty}$ ), then $P$-a.s. the eigenfunctions of $h_{\omega}$ decay as

$$
\left|\psi_{E, \omega}(n)\right| \leq C_{\omega, E, k}\langle n\rangle^{-k}
$$


for any $k>0$. On the other hand, if $j(n)$ decays exponentially (i.e. $\hat{j}(\phi)$ is analytic), it does not follow from our argument that the eigenfunctions of $h_{\omega}$ decay exponentially. To establish such decay using our techniques appears to be difficult technical problem.

If $\hat{j}(\varphi)$ does not satisfy the conditions of Theorem 1.1, we can still prove localization providing random variables $v_{\omega}(n)$ could get large enough. Let

$$
\begin{aligned}
\nu_{0} & \equiv \operatorname{ess.sup}_{\omega}\left|v_{\omega}(n)\right| \\
j_{0} & \equiv \sum|j(n)| \\
\eta_{0} & \equiv \nu_{0}-j_{0}
\end{aligned}
$$

Note that $\nu_{0}$ does not depend on $n$ since the random variables $v_{\omega}(n)$ are identically distributed. If $\eta_{0}>0$, we set $I_{\nu} \equiv\left[-\eta_{0}, \eta_{0}\right]$, otherwise $I_{\nu} \equiv \emptyset$.

Theorem 1.2 Assume that int $\mathcal{V} \neq \emptyset$ and let $\mathcal{J} \equiv I_{\nu} \cup\left(\mathbf{R} \backslash\left(\sigma\left(h_{0}+a_{0}\right)\right)\right.$, where $a_{0} \in \operatorname{int}(\mathcal{V})$.

1. If $\gamma>4$ then $\Sigma_{a c} \cap \mathcal{J}=\emptyset$.

2. If $\gamma>8$ then $\Sigma_{c} \cap \mathcal{J}=\emptyset$.

Remark. The assumption that $\mathcal{V}$ is a compact set is made for convenience reasons, and is not used in the proof of Theorem 1.2, part 2. Thus, whenever $\mathcal{V}$ is unbounded and $\gamma>8, \Sigma_{c}=\emptyset$. Remark 2 after Theorem 1.1 holds also for Theorem 1.2.

Our proofs are based on an approach to localization in $d=1$ pioneered by Simon and Spencer [SS], and further developed in [KMP], [M], [M1], [GJMS]. The principal idea is to show that a particle with energy in a given interval $\mathcal{I}$ has to tunnel through an infinite sequence of "barriers" to reach infinity. These barriers can be the usual potential barriers, as in Theorem 1.2, or the tunneling can be forced due to the gaps in the spectrum of long periodic approximations of $h_{\omega}$, as in Theorem 1.1. In either case, under the conditions of the theorems, we can prove that such barriers exists and that they are effective in preventing tunneling.

Simon and Spencer have discussed the deterministic model $h=h_{0}+v$, where $v$ is a bounded potential. Their result (see (c) in Introduction and Theorem 7.5 in [SS]) can be paraphrased as follows:

Theorem 1.3 (Simon-Spencer) Assume that $\gamma>4$. Let $I_{k}, k \in \mathbf{Z}$, be a sequence of intervals with centers $c_{k}$ and of width $l_{k}$ such that $c_{k} \rightarrow \pm \infty$ and $l_{k} \rightarrow \infty$ as $k \rightarrow \pm \infty$. If $v_{0}$ is a potential and

$$
\max _{n \in I_{k}}\left|v(n)-v_{0}(n)\right| \rightarrow 0
$$

as $k \rightarrow \pm \infty$, then $\sigma_{a c}(h) \subset \sigma\left(h_{0}+v_{0}\right)$.

Our proof of Theorem 1.1, part 1, goes as follows. We show that for each fixed energy $E \in \Sigma$ there is an open interval $\mathcal{I} \ni E$, and a periodic potential $v_{p}$, such that $\mathcal{I} \cap \sigma\left(h_{0}+v_{p}\right)=\emptyset$, 
and that conditions of Theorem 1.3 are satisfied for a.e $\omega$. That is, for a.e. $\omega$ there exists a sequence of intervals $I_{k}(\omega)$ satisfying the conditions of Theorem 1.3 so that (1.5) holds with $v=v_{\omega}$ and $v_{0}=v_{p}$. Then $\Sigma_{a c} \cap \mathcal{I}=\emptyset$, and since $E$ is arbitrary, Theorem 1.1, part 1, follows. We remark that if $h_{0}$ is the usual free Laplacian, a similar proof of absence of a.c. spectrum for one dimensional Anderson model is given in [SS]. Our main contribution here is a novel construction of spectral gaps for periodic approximations of $h_{\omega}$ which is applicable in the long range case. This construction is presented in Section 4.

To idea of Simon and Spencer has been to use trace class perturbations to show the absence of a.c. spectrum. A more detailed analysis is needed to prove localization. Following the ideas of [KMP], [M], [M1], [GJMS], we will prove Theorem 1.1, part 2, by constructing a suitable cluster expansion of the resolvent $\left(h_{\omega}-z\right)^{-1}$ with respect to the intervals $I_{k}(\omega)$. Such an expansion allows for a finer analysis of tunneling. However, we need a more restrictive condition on $\gamma$ to control the convergence of the expansion. Let $m$ be the Lebesgue measure on $\mathbf{R}$. Under the conditions of Theorem 1.1, part 2, we will show that for a.e. $(E, \omega)$ with respect to the product measure $m \otimes P$,

$$
\lim _{\zeta \downarrow 0} \sum_{n \in \mathbf{Z}}\left|\left(\delta_{0},\left(h_{\omega}-E-i \zeta\right)^{-1} \delta_{n}\right)\right|^{2}<\infty
$$

The result then follows from the Simon-Wolff theorem [SW] (for its various reformulations see $[\mathrm{AM}])$.

The proof of Theorem 1.2 follows a similar strategy, except that tunneling is now forced by a trivial gap if $E \notin \sigma\left(h_{0}+a_{0}\right)$, and by potential barriers if $E \in\left[-\eta_{0}, \eta_{0}\right]$.

The results proven here are used in $[\mathrm{JM}]$ to study the propagation properties of surface waves in regions with random boundaries in dimension $d=2$. For additional information on the theory of surface waves and its relation to spectral theory of long range Hamiltonians, we refer the reader to $[\mathrm{JMP}],[\mathrm{G}]$ and $[\mathrm{JL}]$.

The paper is organized as follows. In the next section we collect some preliminary technical results. In Section 3 we study deterministic operators of the form $h_{0}+v$. We prove there our principal technical result, Theorem 3.1, which shows that under suitable assumptions on the existence of tunneling barriers, (1.6) holds in a deterministic setting. In Sections 4 and 5 we study gaps in the spectrum of the operators $h_{0}+v_{p}$, where $v_{p}$ is a periodic potential. Finally, in Section 6 we combine these results with some probabilistic arguments to finish the proofs of Theorems 1.1 and 1.2.

Acknowledgments. We are grateful to B. Simon for many useful discussions. The research of the first author was supported in part by NSERC and of the second by NSF. Part of this work was done while the first author was a visitor at California Institute of Technology. V.J. is grateful to B. Simon and C. Peck for their hospitality. Another part was done during the visit 
of the second author to University of Ottawa which was supported by NSERC.

\section{Preliminaries}

In this section we collect a few technical results which we will use in the sequel. Henceforward we will use normalization $j(0)=0$ in (1.2).

A path $\tau$ connecting $n$ and $m$ is any sequence of sites $\tau=\left(i_{0}, i_{1}, \ldots i_{k}\right)$ such that $i_{0}=n$, $i_{k}=m$. The length of this path is $|\tau|=k$. To the path $\tau$ we associate a sequence of bonds $\tau_{b}=\left(b_{1}, \ldots, b_{k}\right)$, where

$$
b_{1}=\left(i_{0}, i_{1}\right), b_{1}=\left(i_{1}, i_{2}\right), \ldots, b_{k}=\left(i_{k-1}, i_{k}\right)
$$

We write $s \in \tau$ if $s$ is one of the sites of the path $\tau$, and $b=(s, t) \in \tau_{b}$ if $b$ is one of its bonds. We use the shorthand $j(b)=j(s-t)$. Let $h=h_{0}+v$, where $v$ is an arbitrary potential, and

$$
\begin{aligned}
R(z) & =(h-z)^{-1} \\
R(n, m ; z) & =\left(\delta_{n}, R(z) \delta_{m}\right) .
\end{aligned}
$$

Recall that $j_{0}$ is given by (1.4). Let $\delta_{n m}$ be the usual Kronecker symbol.

Proposition 2.1 If $\operatorname{Im}(z)>j_{0}$ then

$$
R(n, m ; z)=-\frac{\delta_{n m}}{z-v(n)}-\sum_{\tau}\left[\prod_{s \in \tau} \frac{1}{z-v(s)}\right] \cdot\left[\prod_{b \in \tau_{b}} j(b)\right],
$$

where the sum is over all paths connecting $n$ and $m$. For each $\varepsilon>0$ the series converges uniformly in the half-plane $\operatorname{Im}(z) \geq j_{0}+\varepsilon$.

Proof: We split the set of paths connecting $n$ and $m$ into the disjoint classes $\mathcal{T}_{k}$ such that $\tau \in \mathcal{T}_{k}$ iff $|\tau|=k$. For any $k$,

$$
\sum_{\tau \in \mathcal{T}_{k}} \prod_{b \in \mathcal{T}_{b}}|j(b)| \leq\left(\sum_{n}|j(n)|\right)^{k}=j_{0}^{k}
$$

and if $\operatorname{Im}(z)>j_{0}$ then

$$
\sum_{k>0} \sum_{\tau \in \mathcal{T}_{k}}\left[\prod_{s \in \mathcal{\tau}}\left|(z-v(s))^{-1}\right|\right] \cdot\left[\prod_{b \in \tau_{b}}|j(b)|\right] \leq \sum_{k>0} \frac{j_{0}^{k}}{|\operatorname{Im}(z)|^{k+1}}<\infty .
$$

Thus, the series (2.7) converges uniformly in the half-plane $\operatorname{Im}(z) \geq j_{0}+\varepsilon$ for any $\varepsilon>0$. Since

$$
\left(h_{0}+v\right) R(z)=I+z R(z)
$$


we get

$$
\left(h_{0} \delta_{n}, R(z) \delta_{m}\right)=\delta_{n m}+(z-v(n)) R(n, m ; z) .
$$

Expanding $h_{0} \delta_{n}$ in the basis $\left\{\delta_{i}\right\}$ we get

$$
R(n, m ; z)=-\frac{\delta_{n m}}{z-v(n)}+\sum_{i} \frac{j(n-i)}{z-v(n)} R(i, m ; z) .
$$

Iterating this formula we derive Relation (2.7).

Proposition 2.1 is known as the path expansion of the resolvent. A similar result holds if the system is restricted to a box. Let $I \subset \mathbf{Z}$ be an arbitrary set, and let $h_{0}^{D}$ be the operator $h_{0}$ restricted to $I$ with Dirichlet boundary condition. This operator is obtained by removing the couplings between the points in $I$ and $\mathbf{Z} \backslash I$, and acts on $l^{2}(I)$ according to the formula

$$
\left(h_{0}^{D} \psi\right)(n)=\sum_{m \in I} j(n-m) \psi(m)
$$

Note that if the support of $\psi \in l^{2}(\mathbf{Z})$ is contained in $I$ then $\left(\psi, h_{0} \psi\right)=\left(\psi, h_{0}^{D} \psi\right)$. It follows that $\sigma\left(h_{0}^{D}\right) \subset \sigma\left(h_{0}\right)$ and $\left\|h_{0}^{D}\right\| \leq j_{0}$. We now define the operator $h_{I}$ on $l^{2}(I)$ by the formula $h_{I}=h_{0}^{D}+v$. We will refer to $h_{I}$ as the restriction of $h=h_{0}+v$ to $I$ with the Dirichlet boundary condition. Let $R_{I}(z)=\left(h_{I}-z\right)^{-1}$. Then for $n, m \in I$,

$$
R_{I}(n, m ; z)=-\frac{\delta_{n m}}{z-v(n)}-\sum_{\tau}\left[\prod_{s \in \mathcal{\tau}} \frac{1}{z-v(s)}\right] \cdot\left[\prod_{b \in \tau_{b}} j(b)\right],
$$

where the sum is over all paths which connect $n$ and $m$ and belong to $I$. If $n$ or $m \notin I$, we set

$$
R_{I}(n, m ; z)=0
$$

In the proofs of Theorems 1.1 and 1.2 we will make use of the following result which is an easy consequence of Corrolary 7.3 in [SS]. We sketch the proof for readers convenience. If $\mathcal{I}=(c, d)$ is an interval in $\mathbf{R}$, we write $I_{\delta}=(c+\delta, d-\delta)$. Recall that $\gamma$ is given by (1.3).

Proposition 2.2 Let $\mathcal{I}$ be an interval such that $\sigma\left(h_{I}\right) \cap \mathcal{I}=\emptyset$ and $l$ be an integer such that $\gamma>l+1$. Then for every $\delta>0$ there is a constant $C_{\delta}$, which depends on $\delta$ only, such that for $E \in \mathcal{I}_{\delta}$,

$$
\left|R_{I}(n, m ; E)\right| \leq C_{\delta}\langle n-m\rangle^{-l}
$$

Proof: We define the operator $x$ on $l^{2}(I)$ by $(x \phi)(n)=n \phi(n)$. Since $\gamma>l+1$, one can show (see Lemma 7.1 in $[\mathrm{SS}]$ ) that the $k$-fold commutator $\left[x, \ldots\left[x, h_{0}\right] \ldots\right]$ is a bounded operator if $1 \leq k \leq l$, and that its norm does not depend on $I$. Using the identity

$$
\left[x, \exp \left(i t h_{I}\right)\right]=i \int_{0}^{t} \exp \left(i s h_{I}\right)\left[x, h_{I}\right] \exp \left(i(t-s) h_{I}\right) d s,
$$


and arguing inductively, one shows that

$$
\left\|\left[x, \ldots\left[x, \exp \left(i t h_{I}\right)\right] \ldots\right]\right\| \leq C(1+|t|)^{k}
$$

for any $1 \leq k \leq l$. Choose now $C_{0}^{\infty}$ function $f$ such that $f=1$ on $\sigma\left(h_{I}\right)$ and $f=0$ on $I_{\delta / 2}$. Let $g_{E}(t)=(t-E)^{-1} f(t)$. Clearly, as long as $E \in \mathcal{I}_{\delta}, f$ can be chosen so that $g_{E}$ is a $C_{0}^{\infty}$ functions whose derivatives have bounds which depend only on $\delta$. Note that $g_{E}\left(h_{I}\right)=\left(h_{I}-E\right)^{-1}$. Since

$$
g_{E}\left(h_{I}\right)=\frac{1}{\sqrt{2 \pi}} \int \widehat{g}_{E}(s) \exp \left(i s h_{I}\right) d s,
$$

it follows that the $k$-fold commutators $\left[x, \ldots\left[x, g_{E}\left(h_{I}\right)\right] \ldots\right]$ are bounded operators if $1 \leq k \leq l$, whose norms have bounds which depend only on $\delta$ (if $E \in \mathcal{I}_{\delta}$ ). Finally, the result follows from the identities

$$
\begin{aligned}
(n-m)^{l}\left(\delta_{n},\left(h_{I}-E\right)^{-1} \delta_{m}\right) & =(n-m)^{l}\left(\delta_{n}, g_{E}\left(h_{I}\right) \delta_{m}\right) \\
& =\left(\delta_{n},\left[x, \ldots\left[x, g_{E}\left(h_{I}\right)\right] \ldots\right] \delta_{m}\right)
\end{aligned}
$$

Recall that $j_{0}$ is given by (1.4). We will also need

Proposition 2.3 Let $l$ an integer such that $\gamma>l+1$ and $\mathcal{I} \subset \mathbf{R}, I \subset \mathbf{Z}$ sets such that for some $\delta>0$

$$
\inf _{n \in I, E \in \mathcal{I}}|v(n)-E| \geq j_{0}+\delta .
$$

Then $\sigma\left(h_{I}\right) \cap \mathcal{I}=\emptyset$, and for all $E \in \mathcal{I}$,

$$
\left|R_{I}(n, m ; E)\right| \leq C_{\delta}\langle n-m\rangle^{-l}
$$

where $C_{\delta}$ depends on $\delta$ only. Furthermore, there is a constant $C$ such that for $\delta>1, C_{\delta}<C / \delta$.

Proof: Since $\left\|h_{0}^{D}\right\| \leq j_{0}$

$$
\sigma\left(h_{I}\right) \subset\left[-j_{0}, j_{0}\right]+\overline{\{v(n): n \in I\}}
$$

see e.g. Lemma 5.3 below. It follows that $\sigma\left(h_{I}\right) \cap \mathcal{I}=\emptyset$. To prove the bound on the resolvent, we consider first the case $n \neq m$. Let $\alpha=j_{0}+\delta$ and $\ell=n-m$. The path expansion of the resolvent $R_{I}$ (recall (2.9)) leads to the bound

$$
\left|R_{I}(n, m ; E)\right| \leq \frac{1}{\alpha} \sum_{k=1}^{\infty} \frac{1}{\alpha^{k}} \sum_{\substack{s_{1}, \ldots s_{k} \in \mathbf{Z} \\ s_{1}+\ldots+s_{k}=\ell}} \prod_{1 \leq i \leq k}\left|j\left(s_{i}\right)\right| .
$$

If

$$
h(\varphi)=\sum_{s} e^{i s \varphi}|j(s)|
$$


then

$$
\sum_{\substack{s_{1}, \ldots, s_{k} \in \mathbf{Z} \\ s_{1}+\ldots+s_{k}=\ell}} \prod_{1 \leq i \leq k}\left|j\left(s_{i}\right)\right|=\frac{1}{2 \pi} \int_{-\pi}^{\pi} e^{-i \varphi \ell} h(\varphi)^{k} d \varphi .
$$

Since $|h(\varphi)| \leq j_{0}<\alpha$, we have that

$$
\left|R_{I}(n, m ; E)\right| \leq \frac{1}{2 \pi \alpha} \sum_{k=1}^{\infty} \int_{-\pi}^{\pi} e^{-i \varphi \ell}\left(\frac{h(\varphi)}{\alpha}\right)^{k} d \varphi=\frac{1}{2 \pi \alpha} \int_{-\pi}^{\pi} e^{-i \varphi \ell} \frac{h(\varphi)}{\alpha-h(\varphi)} d \varphi .
$$

Since the function $h(\varphi) /(\alpha-h(\varphi))$ is $l$-times continuosly differentiable, the result follows from integration by parts. The estimate of $C_{\delta}$ if $\delta>1$ is obvious.

If $n=m$, the argument is simpler, and in fact follows from the observation that if $E \in \mathcal{I}$ then $\operatorname{dist}\left\{E, \sigma\left(h_{I}\right)\right\} \geq \delta$. One can also argue directly:

$$
\begin{aligned}
R_{I}(n, n ; E) & \leq \frac{1}{\alpha}+\sum_{k=2}^{\infty} \frac{1}{\alpha^{k+1}} \sum_{\substack{s_{1}, \ldots s_{k} \in \mathbf{Z} \\
s_{1}+\ldots+s_{k}=0}} \prod_{1 \leq i \leq k}\left|j\left(s_{i}\right)\right| \\
& \leq \frac{1}{\alpha}+\sum_{k=1}^{\infty} \frac{1}{\alpha^{k+1}}\left(\sum_{s}|j(s)|\right)^{k}=\frac{1}{\delta} .
\end{aligned}
$$

We will also make use of the following two versions of the well-known Kolmogorov inequality. For the proofs we refer the reader to [M1], [GJMS]. The history of the Kolomogorov inequality is discussed in [A]. In the sequel $|A|$ stands for the Lebesgue measure of the set $A$.

Proposition 2.4 Let $\alpha_{1}, \ldots, \alpha_{n}$ and $\lambda_{1}, \ldots, \lambda_{n}$ be real numbers such that $\sum\left|\alpha_{k}\right| \leq 1$. Let $h$ and $f$ be functions defined by

$$
h(\lambda) \equiv \sum_{k=1}^{n} \frac{\alpha_{k}}{\lambda-\lambda_{k}}, \quad f(\lambda) \equiv \sum_{k=1}^{n} \frac{\left|\alpha_{k}\right|}{\left(\lambda-\lambda_{k}\right)^{2}},
$$

where $\lambda \in \mathbf{R}$. Then

$$
\begin{aligned}
|\{\lambda:|h(\lambda)|>M\}| & \leq 2 / M, \\
|\{\lambda: f(\lambda)>M\}| & \leq 4(n / M)^{1 / 2} .
\end{aligned}
$$

The final technical result we need is:

Proposition 2.5 Let $I_{\ell}$ be a sequence of finite intervals such that $I_{\ell} \uparrow \mathbf{Z}$ as $\ell \rightarrow \infty$, and let $\mathcal{M}$ be a measurable set. Then, $\forall n \in \mathbf{Z}$ and a.e. $E \in \mathcal{M}$,

$$
\lim _{\zeta \rightarrow 0} \sum_{m \in \mathbf{Z}}\left|\left(\delta_{n},(h-E-i \zeta)^{-1} \delta_{m}\right)\right|^{2} \leq \liminf _{\ell \rightarrow \infty} \sum_{m \in I_{\ell}}\left|\left(\delta_{n},\left(h_{I_{\ell}}-E\right)^{-1} \delta_{m}\right)\right|^{2} .
$$


Proof: Let $\mathcal{M}^{\prime}=\mathcal{M} \backslash\left(\cup_{\ell} \sigma\left(h_{I_{\ell}}\right)\right)$. Since each $h_{I_{\ell}}$ has a discrete spectrum, $\left|\mathcal{M}^{\prime}\right|=|\mathcal{M}|$. We denote by $\mu_{n}^{\ell}$ and $\mu_{n}$ the spectral measures associated to the vector $\delta_{n}$ and the operators $h_{I_{\ell}}$ and $h$. For each $E \in \mathcal{M}^{\prime},\left(h_{I_{\ell}}-E\right)^{-1}$ is well-defined and

$$
\sum_{m \in I_{\ell}}\left|\left(\delta_{n},\left(h_{I_{\ell}}-E\right)^{-1} \delta_{m}\right)\right|^{2}=\int \frac{d \mu_{n}^{\ell}(\lambda)}{|\lambda-E|^{2}} .
$$

Thus,

$$
\begin{aligned}
\lim _{\zeta \rightarrow 0} \sum_{m \in \mathbf{Z}}\left|\left(\delta_{n},(h-E-i \zeta)^{-1} \delta_{m}\right)\right|^{2} & =\lim _{\zeta \rightarrow 0} \int \frac{d \mu_{n}(\lambda)}{|\lambda-E|+\zeta^{2}} \\
& =\lim _{\zeta \rightarrow 0} \lim _{\ell \rightarrow \infty} \int \frac{d \mu_{n}^{\ell}(\lambda)}{|\lambda-E|^{2}+\zeta^{2}} \\
& \leq \liminf _{\ell \rightarrow \infty} \sum_{m \in I_{\ell}}\left|\left(\delta_{n},\left(h_{I_{\ell}}-E\right)^{-1} \delta_{m}\right)\right|^{2}
\end{aligned}
$$

\section{The main theorem}

Let $h_{0}$ be given by (1.2), $v$ be a potential, and $h=h_{0}+v$. We will use freely the notation introduced in the previous section. In this section we prove

Theorem 3.1 Assume that (1.3) holds for some $\gamma>8$. Let $\mathcal{I}=(c, d)$ be an open interval and $a \geq 2$ an integer. Assume that there exists an integer $N \geq 0$ such that, $\forall n>0$, the intervals

$$
\pm\left[a^{N+n}+1, a^{N+n+1}-1\right]
$$

contain sub-intervals $I_{ \pm n}$ of length $l_{ \pm n} \geq n$ such that

$$
\sigma\left(h_{I_{ \pm n}}\right) \cap \mathcal{I}=\emptyset
$$

Then for a.e. $E \in \mathcal{I}$ with respect to the Lebesgue measure,

$$
\lim _{\zeta \downarrow 0} \sum_{m \in \mathbf{Z}}\left|\left(\delta_{0},(h-E-i \zeta)^{-1} \delta_{m}\right)\right|^{2}<\infty
$$

Note that it follows from (3.12) and Proposition 2.2 that for all $E \in \mathcal{I}_{\delta_{0}} \equiv\left(c+\delta_{0}, d-\delta_{0}\right)$,

$$
\left|R_{I_{n}}\left(k, k^{\prime} ; E\right)\right| \leq C_{\delta_{0}}\left\langle k-k^{\prime}\right\rangle^{-l}
$$

where $l$ is an integer such that $\gamma>l+1$ (e.g. $l=7$ ), and the constant $C_{\delta_{0}}$ depends only on $\delta_{0}$. In the sequel we fix small $\delta_{0}>0$ and establish Relation (3.13) for a.e. $E \in \mathcal{I}_{\delta_{0}}$. Since $\delta_{0}>0$ is arbitary, this suffices.

We begin by introducing several sequences of intervals which will play an important role in the sequel. Let the $I_{n}$ 's be as in the theorem, $I_{n} \equiv\left[a_{n}, b_{n}\right]$ and $l_{n}=\left|a_{n}-b_{n}\right|+1$. Let $M_{0}=\left[a_{-1}, b_{1}\right]$. 
For $n>0$, we set $M_{n}=\left[a_{n}, b_{n+1}\right]$, and for $n<0, M_{n}=\left[a_{n-1}, b_{n}\right]$. We will refer to the intervals $M_{n}$ as the main blocks. Let $\Delta_{0}=\left[b_{-1}, a_{1}\right]$. For $n>0$, we set $\Delta_{n}=\left[b_{n}, a_{n+1}\right]$, and for $n<0$, $\Delta_{n}=\left[b_{n-1}, a_{n}\right]$. In the sequel we will refer to the $I_{n}$ 's as the black blocks and to $\Delta_{n}$ 's as the white blocks. Note that for $n>0$,

$$
M_{n}=I_{n} \cup \Delta_{n} \cup I_{n+1}
$$

A similar relation holds for $n<0$.

The strategy of our argument is the following. The black blocks are long barriers. Although we do not have any information about the values of the potential within the white blocks, we know that these blocks are not "too long." We will construct a suitable expansion of the resolvent $(h-z)^{-1}$ in terms of the main blocks $M_{n}$. We then use the decomposition (3.15) and tunneling estimates to further refine this expansion, and to establish (3.13).

We denote by $h_{M_{n}}$ the restriction of $h$ to $M_{n}$ with Dirichlet boundary condition. Let $R_{M_{n}}(z)$ be the resolvent of $h_{M_{n}}$ and $R_{M_{n}}(p, q ; z)$ its matrix elements. We first collect some a priori estimates on $R_{M_{n}}$. Let

$$
x_{n}^{(1)}=a_{n}, x_{n}^{(2)}=b_{n}, x_{n}^{(3)}=a_{n+1}, x_{n}^{(4)}=b_{n+1} .
$$

Recall that $\gamma>8$ and that $\langle x\rangle=\left(1+x^{2}\right)^{1 / 2}$. Throughout, we will freely use the convention $(2.10)$.

Proposition 3.2 Let $\delta>0$ be such that $\gamma / 4>2(1+\delta)$. Then for every $\varepsilon>0$ there is a set $\mathcal{M}_{\varepsilon} \subset \mathbf{R}$ such that:

1. $\left|\mathbf{R} \backslash \mathcal{M}_{\varepsilon}\right|=0$.

2. For each $E \in \mathcal{M}_{\varepsilon}$ there is a positive integer $n_{E, \varepsilon}$ such that for $|n| \geq n_{E, \varepsilon}$ the following estimates hold:

$$
\begin{aligned}
\max _{i, j}\left|R_{M_{n}}\left(x_{n}^{(i)}+p, x_{n}^{(j)}+q ; E\right)\right| & \leq \varepsilon\langle n\rangle^{1+\delta}\langle p\rangle^{1+\delta}\langle q\rangle^{1+\delta}, \\
\max _{i} \sum_{q \in M_{n}}\left|R_{M_{n}}\left(x_{n}^{(i)}+p, q ; E\right)\right|^{2} & \leq\left(\left|M_{n}\right|+1\right)\langle n\rangle^{2(1+\delta)}\langle p\rangle^{2(1+\delta)}, \\
\max _{|p-q|>l_{n} / 2}\left|R_{I_{n}}(p, q ; E)\right| & <\varepsilon\langle p-q\rangle^{-6} .
\end{aligned}
$$

Proof: Let $L_{n}=\left|M_{n}\right|+1$. Then

$$
R_{M_{n}}\left(x_{n}^{(i)}+p, x_{n}^{(j)}+q ; E\right)=\sum_{k=1}^{L_{n}} \frac{\phi_{k}\left(x_{n}^{(i)}+p\right) \phi_{k}\left(x_{n}^{(j)}+q\right)}{E-E_{k}},
$$

where $\phi_{k}$ are eigenfunctions and $E_{k}$ eigenvalues of $h_{M_{n}}$. Let

$$
A_{n}(p, q)=\left\{E:\left|R_{M_{n}}\left(x_{n}^{(i)}+p, x_{n}^{(j)}+q ; E\right)\right|>\frac{\varepsilon}{8}\langle n\rangle^{1+\delta}\langle p\rangle^{1+\delta}\langle q\rangle^{1+\delta} \text { for } 1 \leq i, j \leq 4\right\} .
$$


Note that

$$
\sum_{k=1}^{L_{n}}\left|\phi_{k}\left(x_{n}^{(i)}+p\right)\right| \cdot\left|\phi_{k}\left(x_{n}^{(j)}+q\right)\right| \leq\left(\sum_{k=1}^{L_{n}}\left|\phi_{k}\left(x_{n}^{(i)}+p\right)\right|^{2}\right)^{1 / 2}\left(\sum_{k=1}^{L_{n}}\left|\phi_{k}\left(x_{n}^{(j)}+q\right)\right|^{2}\right)^{1 / 2}=1 .
$$

It follows from Proposition 2.4 that $\left|A_{n}(p, q)\right| \leq[\langle n\rangle\langle p\rangle\langle q\rangle]^{-1-\delta} / \varepsilon$. Since

$$
\sum_{n} \sum_{p, q}\left|A_{n}(p, q)\right|<\infty
$$

it follows from the Borel-Cantelli lemma that there exists a measurable set $\mathcal{M}_{\varepsilon}^{\prime}$ such that 1 . and 2., (3.16) hold.

We now consider Relation (3.17). Note first that

$$
\sum_{q \in M_{n}}\left|R_{M_{n}}\left(x_{n}^{(i)}+p, q ; E\right)\right|^{2}=\sum_{k=1}^{L_{n}} \frac{\left|\phi_{k}\left(x_{n}^{(i)}+p\right)\right|^{2}}{\left|E-E_{k}\right|^{2}}
$$

It follows again from Proposition 2.4 and the Borel-Cantelli lemma that there exists a measurable set $\mathcal{M}^{\prime \prime}$ (which does not depend on $\varepsilon$ ) such that 1. and 2., (3.17) hold. Taking $\mathcal{M}_{\varepsilon}=\mathcal{M}_{\varepsilon}^{\prime} \cap \mathcal{M}^{\prime \prime}$, we deduce that 1., and 2, (3.16), (3.17) hold.

Recall that $C_{\delta_{0}}$ is given by (3.13). Let $q_{\varepsilon}$ be an integer such that

$$
n>q_{\varepsilon} \Rightarrow 2 C_{\delta_{0}} / n<\varepsilon
$$

Clearly, we may assume that $n_{E, \varepsilon}$ is chosen in such a way that $n_{E, \varepsilon}>q_{\varepsilon}$, and thus that (3.16)(3.18) hold for each $E \in \mathcal{M}_{\varepsilon}$ and $|n|>n_{E, \varepsilon}$.

Remark 1. The various parametrizations $x_{n}^{(i)}$ in the previous lemma are introduced for later convenience.

Remark 2. Note that $n_{E, \varepsilon}$ is not specified uniquely. To avoid some ambiguites, for given $\varepsilon>0$ and $E \in \mathcal{M}_{\varepsilon}$ we define $n_{E, \varepsilon}$ as the smallest positive integer such that (3.16)-(3.18) hold for all $|n| \geq n_{E, \varepsilon}$.

Proposition 3.2 gives information on the resolvent matrix elements of $R_{M_{n}}$ starting with a sufficiently large index $n$ which depends on the energy. To circumvent some difficulties which arise from this $E$-dependence, we introduce the sets

$$
\mathcal{M}_{k, \varepsilon}=\bigcup_{j=0}^{k}\left\{E: E \in \mathcal{I}_{\delta_{0}} \text { and } n_{E, \varepsilon}=j\right\}
$$

Since $R_{M_{n}}(s, t ; E)$ are Lebesgue measurable functions of $E$, the sets $\mathcal{M}_{k, \varepsilon}$ are measurable. Clearly, if $i>k$ then $\mathcal{M}_{k, \varepsilon} \subset \mathcal{M}_{i, \varepsilon}$ Furthermore, it follows from Proposition 3.1 that for each $\varepsilon>0$, $\cup_{k \geq 0} \mathcal{M}_{k, \varepsilon}$ is of full measure in $\mathcal{I}_{\delta_{0}}$. Note that some of the sets $\mathcal{M}_{k, \varepsilon}$ might be empty. However, 
for each $\varepsilon>0$ there is $k(\varepsilon)>0$ such that $\mathcal{M}_{k, \varepsilon} \neq \emptyset$ if $k>k(\varepsilon)$. Let $C$ be a constant from Proposition 2.3 and let

$$
L=\max \{|c|,|d|\}+j_{0}+C / \varepsilon,
$$

(recall that $\mathcal{I}=(c, d)$ ). For given $k$ and $\varepsilon$, we introduce an auxiliary potential $v_{k, \varepsilon}$ by the formula

$$
v_{k, \varepsilon}(n)= \begin{cases}L & \text { if } n \in M_{s},|s| \leq k \\ v(n) & \text { if } n \in M_{s},|s|>k\end{cases}
$$

The reasons for introducing this auxiliary potential are the following:

a) If $E \in \mathcal{M}_{k, \varepsilon}$ and $v$ is replaced by $v_{k, \varepsilon}$ then the inequalites (3.16) and (3.17) hold for all $n$.

b) If $|n| \leq k$ then it follows from Proposition 2.3 and the choice of $L$ that the inequality (3.18) holds for all $p, q \in I_{n}$.

Let

$$
J_{\ell} \equiv \bigcup_{j,|j| \leq \ell} M_{j}
$$

We denote by $h_{\ell, k, \varepsilon}$ the operator $h_{0}+v_{k, \varepsilon}$ restricted to $J_{\ell}$ with Dirichlet boundary condition. We will prove below the following result.

Proposition 3.3 There exists $\varepsilon_{0}>0$ such that for $k>k\left(\varepsilon_{0}\right), E \in \mathcal{M}_{k, \varepsilon_{0}}$, and $i \in \cup_{s=-k}^{k} M_{s}$,

$$
\limsup _{\ell \rightarrow \infty} \sum_{m \in J_{\ell}}\left|\left(\delta_{i},\left(h_{\ell, k, \varepsilon_{0}}-E\right)^{-1} \delta_{m}\right)\right|^{2}<\infty
$$

Let us show how Relation (3.13) (for $n=0$ ) follows from this proposition. Denote for the moment by $R_{k, \varepsilon_{0}}$ the resolvent of the operator $h_{0}+v_{k, \varepsilon_{0}}$. It then follows from Propositions 2.5 and 3.3 that for $E \in \mathcal{M}_{k, \varepsilon_{0}}$ and $i \in \cup_{s=-k}^{k} M_{s}$,

$$
\lim _{\zeta \rightarrow 0} \sum_{m \in \mathbf{Z}}\left|R_{k, \varepsilon_{0}}(i, m ; E+i \zeta)\right|^{2} \leq C_{i, k, \varepsilon_{0}}<\infty
$$

Furthermore, it follows from the resolvent identity that

$$
R(0, m ; E+i \zeta)=R_{k, \varepsilon_{0}}(0, m ; E+i \zeta)+\sum_{i \in M_{s},|s| \leq k}(L-v(i)) R(0, i ; E+i \zeta) R_{k, \varepsilon_{0}}(i, m ; E+i \zeta) .
$$

Since for a.e. $E \in \mathbf{R}, \lim _{\zeta \rightarrow 0} R(0, i ; E+i \zeta)$ exists and is finite, we derive that for a.e. $E \in \mathcal{M}_{k, \varepsilon_{0}}$,

$$
|R(0, m ; E+i \zeta)|^{2} \leq C_{E} \sum_{i \in M_{s},|s| \leq k}\left|R_{k, \varepsilon_{0}}(i, m ; E+i \zeta)\right|^{2}
$$

This inequality and (3.20) yield Relation (3.13) for $n=0$ and for a.e. $E \in \cup_{k} M_{k, \varepsilon_{0}}$.

The rest of this section is devoted to the proof of Proposition 3.3.

Notation. In the sequel we will denote by the same letter $C$ various constants which depend 
only on $C_{\delta_{0}}$ in (3.14). The values of these constants may change from estimate to estimate. Furthermore, we will drop the subscripts $k$ and $\varepsilon$ in the sequel whenever there is no danger of confusion. For example, we denote by $R_{\ell}(n, m ; z)$ the matrix elements of the resolvent $\left(h_{\ell, k, \varepsilon}-\right.$ $z)^{-1}$, etc.

We will prove Proposition 3.3 in the case where $i=0$. Also, we will assume that $a=2$ in (3.11). A similar argument applies to the other values of $i$ and $a$.

Let $\ell>0$ be given. Our first goal is to develop a suitable expansion of the matrix resolvent element $R_{\ell}(0, m ; z)$ with respect to $R_{M_{s}}$. Let $\tau$ be any path in the expansion (2.9) which connects 0 and $m, \tau=\left(0, n_{1}, n_{2}, \ldots, n_{k}, m\right)$. To such a path we associate a sequence of bonds $\left(b_{1}, \ldots, b_{l}\right)$ and a sequence of blocks $\left(M_{s_{1}}, \ldots, M_{s_{l}}\right)$ in the following way. Let $n_{k_{1}}$ be the first of the $n_{l}$ 's which is not in the block $M_{0}$. Then let $b_{1}=\left(n_{k_{1}-1}, n_{k_{1}}\right)$. We denote the block to which $n_{k_{1}}$ belongs by $M_{s_{1}}$. Let $n_{k_{2}}$ be the first of the $n_{l}$ 's, for $l>k_{1}$, which is not in $M_{s_{1}}$, and let $b_{2}=\left(n_{k_{2}-1}, n_{k_{2}}\right)$. We denote the block to which $n_{k_{2}}$ belongs by $M_{s_{2}}$. If $n_{k_{2}} \in M_{s} \cap M_{t}$ then, by definition, $s_{2}=\min \{s, t\}$ if $s, t \geq 0$, and $s_{2}=\max \{s, t\}$ if $s, t \leq 0$. We now continue inductively. It is helpful to invoke the following picture. The path $\tau$ starts in the block $M_{0}$, and wanders for some time within this block. It then leaves $M_{0}$ and jumps to a different block $M_{s_{1}}$. In the bond $b_{1}$ we record the site $n_{k_{1}-1} \in M_{0}$ at which the path takes off, and the site $n_{k_{1}} \in M_{s_{1}}$ at which it lands. The path now wanders through $M_{s_{1}}$ and then jumps to $M_{s_{2}}$, etc. The last bond $b_{l}=\left(n_{k_{l-1}}, n_{k_{l}}\right)$ corresponds to the last entry into the block $M_{s_{l}} \equiv M_{n_{0}}$ which contains $m$. Since neighboring blocks intersect, the paths can land at the site which belongs simultaneously to two blocks; in this case, by definition, we say that the path landed in the block which is closer to 0. Clearly, the sequences $\left\{b_{i}\right\}$ and $\left\{M_{s_{i}}\right\}$ do not uniquely determine the path: great many paths $\tau$ will determine the same sequences of blocks. Note that $\left\{b_{i}\right\}$, however, uniquely determines $\left\{M_{s_{i}}\right\}$. Let $\mathcal{B}$ be the set of all sequences of bonds $\tau_{b}=\left\{b_{i}\right\}$ obtained in the above way.

Regrouping the elements in the expansion (2.9) we get

$$
\begin{gathered}
R_{\ell}(0, m ; z)=\delta_{0 m} /(v(0)-z)+\sum_{\tau_{b} \in \mathcal{B}} R_{M_{0}}\left(0, n_{k_{1}-1} ; z\right) j\left(n_{k_{1}-1}-n_{k_{1}}\right) R_{M_{s_{1}}}\left(n_{k_{1}}, n_{k_{2}-1} ; z\right) \ldots \\
\ldots R_{M_{s_{l-1}}}\left(n_{k_{l-1}}, n_{k_{l}-1} ; z\right) j\left(n_{k_{l}-1}-n_{k_{l}}\right) R_{M_{n_{0}}}\left(n_{k_{l}}, m ; z\right) .
\end{gathered}
$$

At this point, of course, this relation holds only for $\operatorname{Im}(z)>j_{0}$. However, for any $z \in \mathbf{C}$, if the series on the right hand side converges absolutely then its sum is $R(0, m ; z)$. To show this, for $z \in \mathbf{C}$ we define

$$
\begin{gathered}
\mathcal{R}_{\ell}(0, m ; z)=\delta_{0 m} /(v(0)-z)+\sum_{\tau_{b} \in \mathcal{B}} R_{M_{0}}\left(0, n_{k_{1}-1} ; z\right) j\left(n_{k_{1}-1}-n_{k_{1}}\right) R_{M_{s_{1}}}\left(n_{k_{1}}, n_{k_{2}-1} ; z\right) \ldots \\
\ldots R_{M_{s_{l-1}}}\left(n_{k_{l-1}}, n_{k_{l}-1} ; z\right) j\left(n_{k_{l}-1}-n_{k_{l}}\right) R_{M_{n_{0}}}\left(n_{k_{l}}, m ; z\right) .
\end{gathered}
$$

whenever the sum converges absolutely. We then have 
Proposition 3.4 If $z \in \mathbf{C}$ and if $\mathcal{R}(0, m ; z)$ is defined for all $m \in J_{\ell}$, then $z \notin \sigma\left(h_{\ell}\right)$ and $R_{\ell}(0, m ; z)=\mathcal{R}_{\ell}(0, m ; z)$.

Remark. In the sequel, we will apply this proposition in the case $z=E \in \mathbf{R}$.

Proof: If the series (3.21) converges absolutely, the various sums can be interchanged, and one easily shows that the vector $\mathcal{R}_{\ell}(0, \cdot ; z) \in l^{2}\left(J_{\ell}\right)$ satisfies the equation

$$
\sum_{n \in J_{\ell}} j(n-m) \mathcal{R}_{\ell}(0, n ; z)+\left(v_{k, \varepsilon}(m)-z\right) \mathcal{R}_{\ell}(0, m ; z)=\delta_{0 m}, \quad m \in J_{\ell}
$$

However, if this equation has a solution then $z \notin \sigma\left(h_{\ell}\right)$, the solution is unique and is equal to $R_{l}(0, m ; z) . \square$.

We proceed to prove the following statement: There exists $\varepsilon_{0}>0$ such that for $k>k\left(\varepsilon_{0}\right)$ and $E \in \mathcal{M}_{k, \varepsilon_{0}}$, the formal series (3.21) converges absolutely and

$$
\sum_{m \in J_{\ell}}\left|\mathcal{R}_{\ell}(0, m ; E)\right|^{2} \leq C<\infty
$$

where the constant $C$ does not depend on $\ell$. Proposition 3.3 then follows from Proposition 3.4.

Let us consider a typical term in the formal expansion (3.21):

$$
R_{M_{s_{i-1}}}\left(n_{k_{i-1}}, n_{k_{i}-1} ; E\right) j\left(n_{k_{i}-1}-n_{k_{i}}\right) R_{M_{s_{i}}}\left(n_{k_{i}}, n_{k_{i+1}-1} ; E\right)
$$

For notational convenience, our analysis of such terms is based on case by case analysis, depending on the arrangment of the blocks $M_{s_{i-2}}, M_{s_{i-1}}, M_{s_{i}}$ and $M_{s_{i+1}}$. There are $4 !=24 \mathrm{such}$ arrangments. We will consider only the arrangment $s_{i+1}>s_{i}>s_{i-1}>s_{i-2}>0$. After this case is analyzed, the reader can easily convince himself that one argues similarly in all the other cases. We denote by $d\left(s_{i-1}, s_{i}\right)$ the distance between the blocks $M_{s_{i-1}}$ and $M_{s_{i}}$. Clearly, if $s_{i}=s_{i-1}+1$, then $d\left(s_{i-1}, s_{i}\right)=0$. In the sequel we fix $\varepsilon>0$ and $k>k(\varepsilon)$, and proceed to obtain a suitable estimate on

$$
R_{M_{s_{i-1}}}\left(n_{k_{i-1}}, n_{k_{i}-1} ; E\right) j\left(n_{k_{i}-1}-n_{k_{i}}\right)
$$

for $E \in \mathcal{M}_{k, \varepsilon}$. Even after specifying the arrangment of the main blocks, our argument is based on the case by case analysis, depending whether the sites $n_{k_{i-1}}, n_{k_{i}-1}$ belong to white or black blocks. Recall that $M_{s_{i-1}}=I_{s_{i-1}} \cup \Delta_{s_{i-1}} \cup I_{s_{i-1}+1}$. The following cases have to be considered:

1. $n_{k_{i-1}}, n_{k_{i}-1} \in \Delta_{s_{i-1}}$.

2. $n_{k_{i-1}} \in \Delta_{s_{i-1}}, n_{k_{i}-1} \in I_{s_{i-1}+1}$.

3. $n_{k_{i-1}}, n_{k_{i}-1} \in I_{s_{i-1}+1}$.

4. $n_{k_{i-1}} \in \Delta_{s_{i-1}}, n_{k_{i}-1} \in I_{s_{i-1}}$.

5. $n_{k_{i-1}} \in I_{s_{i-1}+1}, n_{k_{i}-1} \in \Delta_{s_{i-1}}$. 
6. $n_{k_{i-1}} \in I_{s_{i-1}+1}, n_{k_{i}-1} \in I_{s_{i-1}}$.

Case 1. Recall that $\Delta_{s_{i-1}}=\left[b_{s_{i-1}}, a_{s_{i-1}+1}\right]$. It follows from (1.3) that

$$
\left|j\left(n_{k_{i}-1}-n_{k_{i}}\right)\right| \leq C\left[\left\langle n_{k_{i}-1}-a_{s_{i-1}+1}\right\rangle\left\langle a_{s_{i-1}+1}-b_{s_{i-1}+1}\right\rangle\left\langle d\left(s_{i-1}, s_{i}\right)\right\rangle\left\langle n_{k_{i}}-b_{s_{i}}\right\rangle\right]^{-\gamma / 4},
$$

where $C$ is a universal constant. The last term in the product, $\left\langle n_{k_{i}}-b_{s_{i}}\right\rangle^{-\gamma / 4}$, is used in estimating the term in the expansion which follows after (3.23); in estimating (3.23) we will make use of the similar term which arises in the estimation of $j\left(n_{k_{i-2}}-n_{k_{i}-1}\right)$, namely $\left\langle n_{k_{i-1}}-b_{s_{i-1}}\right\rangle^{-\gamma / 4}$. With this convention, it follows from Proposition 3.2 that for $E \in \mathcal{M}_{k, \varepsilon}$,

$$
\left|R_{M_{s_{i-1}}}\left(n_{k_{i-1}}, n_{k_{i}-1} ; E\right)\right| \leq \varepsilon\left[\left\langle s_{i-1}\right\rangle\left\langle n_{k_{i-1}}-b_{s_{i-1}}\right\rangle\left\langle n_{k_{i}-1}-a_{s_{i-1}+1}\right\rangle\right]^{1+\delta}
$$

Since $l_{s_{i-1}+1} \geq\left|s_{i-1}\right|$ and $\gamma / 4>2(1+\delta)$, we get that

$$
\left|R_{M_{s_{i-1}}}\left(n_{k_{i-1}}, n_{k_{i}-1} ; E\right)\right| \leq C \varepsilon\left[\left\langle s_{i-1}\right\rangle\left\langle n_{k_{i-1}}-b_{s_{i-1}}\right\rangle\left\langle n_{k_{i}-1}-a_{s_{i-1}+1}\right\rangle\right]^{-1-\delta}\left\langle d\left(s_{i-1}, s_{i}\right)\right\rangle^{-\gamma / 4},
$$

for some constant $C$. Thus, for $E \in M_{k, \varepsilon}$,

$$
\sum_{n_{k_{i-1}}, n_{k_{i}-1} \in \Delta_{s_{i}-1}}\left|R_{M_{s_{i}}}\left(n_{k_{i-1}}, n_{k_{i}-1} ; E\right)\right| \cdot\left|j\left(n_{k_{i}-1}-n_{k_{i}}\right)\right| \leq C \varepsilon\left\langle s_{i_{-1}}\right\rangle^{-1-\delta}\left\langle d\left(s_{i-1}, s_{i}\right)\right\rangle^{-\gamma / 4}
$$

Case 2. The critical input in deriving estimate (3.24) is that the path jumps over the long black block $I_{s_{i-1}+1}$. If $n_{k_{i}-1} \in I_{s_{i-1}+1}$ and the sum is over $n_{k_{i}-1}$ such that

$$
n_{k_{i}-1}-a_{s_{i-1}+1} \leq 3 l_{s_{i-1}+1} / 4
$$

then the same argument (with a change of the constant, of course) yields the estimate (3.24). We have choosen the constant 3/4 for definiteness; any $0<\epsilon<1$ will do as well. Difficulties arise if $n_{k_{i}-1}$ is close to $b_{s_{i-1}+1}$; in this case, the jump $M_{s_{i-1}} \rightarrow M_{s_{i}}$ could be very short and we cannot use the previous arguments. So we now assume that

$$
n_{k_{i}-1}-a_{s_{i-1}+1}>3 l_{s_{i-1}+1} / 4 \text {. }
$$

In this case we use the formula

$$
R_{M_{s_{i-1}}}\left(n_{k_{i-1}}, n_{k_{i}-1} ; E\right)=\sum_{\substack{r \in I_{s_{i-1} \cup \Delta_{s_{i-1}}} \\ t \in I_{s_{i-1}+1}}} R_{M_{s_{i-1}}}\left(n_{k_{i-1}}, r ; E\right) j(r-t) R_{I_{s_{i-1}+1}}\left(t, n_{k_{i}-1} ; E\right) .
$$

We will make use of the following two elementary estimates:

$$
\begin{aligned}
\left|j\left(n_{k_{i}-1}-n_{k_{i}}\right)\right| & \leq C\left[\left\langle n_{k_{i}-1}-b_{s_{i-1}+1}\right\rangle\left\langle d\left(s_{i-1}, s_{i}\right)\right\rangle\left\langle n_{k_{i}}-b_{s_{i}}\right\rangle\right]^{-\gamma / 3} \\
|j(r-t)| & \leq C\left[\left\langle r-a_{s_{i-1}+1}\right\rangle\left\langle t-a_{s_{i-1}+1}\right\rangle\right]^{-\gamma / 2}
\end{aligned}
$$


It now follows from Propositions 3.2 that for $E \in M_{k, \varepsilon}$ (recall a) stated before Proposition 3.3)

$$
\left|R_{M_{s_{i-1}}}\left(n_{k_{i-1}}, r ; E\right)\right| \leq \varepsilon\left[\left\langle s_{i-1}\right\rangle\left\langle n_{k_{i-1}}-b_{s_{i-1}}\right\rangle\left\langle r-a_{s_{i-1}}\right\rangle\right]^{1+\delta} .
$$

We now again drop the last term in in the first Equation in (3.28) and use the similar term arising from $j\left(n_{k_{i-2}}-n_{k_{i-1}}\right)$. With this convention and arguing as before, we deduce

$$
\sum_{\substack{n_{k_{i-1}} \in \Delta_{s_{i-1}}>3 l_{s_{i-1} / 4} \\ n_{k_{i}-1} \in I_{s_{i}}, n_{k_{i}-1}-a_{s_{i-1}+1} \mid>l_{s}}}\left|R_{M_{s_{i-1}}}\left(n_{k_{i-1}}, n_{k_{i}-1} ; E\right)\right| \cdot\left|j\left(n_{k_{i}-1}-n_{k_{i}}\right)\right| \leq C \varepsilon\left\langle s_{i-1}\right\rangle^{1+\delta}\left\langle d\left(s_{i-1}, s_{i}\right)\right\rangle^{-\gamma / 4} \mathcal{S}
$$

where

$$
\mathcal{S} \equiv \max _{\left|n_{k_{i}-1}-a_{s_{i-1}+1}\right|>3 l_{s_{i-1}+1} / 4} \sum_{t \in I_{s_{i-1}+1}}\left\langle t-a_{s_{i-1}+1}\right\rangle^{-\gamma / 2}\left|R_{I_{s_{i-1}+1}}\left(t, n_{k_{i}-1} ; E\right)\right| .
$$

We break the sum over $t$ into the regions where $t-a_{s_{i-1}+1} \leq l_{s_{i-1}+1} / 4$ and $t-a_{s_{i-1}+1}>l_{s_{i-1}+1} / 4$. To bound the first sum, we use the bound (3.18) and (3.27) and for the second sum we use that $l_{s_{i-1}+1}>\left|s_{i-1}\right|$. In this way, we arrive at the estimate $\mathcal{S} \leq C\left\langle s_{i-1}\right\rangle^{-2(1+\delta)}$. Combining now the estimates for the cases $(3.26)$ and $(3.27)$ we derive an estimate analogous to (3.25):

$$
\sum_{\substack{n_{k_{i-1}} \in \Delta_{s_{i-1}} \\ n_{k_{i}-1} \in I_{s_{i-1}+1}}}\left|R_{M_{s_{i-1}}}\left(n_{k_{i-1}}, n_{k_{i}-1} ; E\right)\right| \cdot\left|j\left(n_{k_{i}-1}-n_{k_{i}}\right)\right| \leq C \varepsilon\left\langle s_{i-1}\right\rangle^{-1-\delta}\left\langle d\left(s_{i-1}, s_{i}\right)\right\rangle^{-\gamma / 4} .
$$

This concludes the discussion of the Case 2.

Case 3. Assume now that $n_{k_{i-1}}, n_{k_{i}-1} \in I_{s_{i-1}+1}$. Again, we encounter difficulties only if $n_{k_{i}-1}-$ $a_{s_{i-1}+1}>3 l_{s_{i-1}+1} / 4$. In this case we use the formula

$$
\begin{aligned}
& R_{M_{s_{i-1}}}\left(n_{k_{i-1}}, n_{k_{i}-1} ; E\right)=R_{I_{s_{i-1}+1}}\left(n_{k_{i-1}}, n_{k_{i}-1} ; E\right)+ \\
& \sum_{\substack{r \in I_{s_{i-1}+1} \\
t \in \Delta_{s_{i-1} \cup I_{s_{i-1}+1}}}} R_{I_{s_{i-1}+1}}\left(n_{k_{i-1}}, r ; E\right) j(r-t) R_{M_{s_{i-1}}}\left(t, n_{k_{i}-1} ; E\right) .
\end{aligned}
$$

If $E \in \mathcal{M}_{k, \varepsilon}$ and $s_{i-1}+1 \leq k$, then it follows from the definition of $v_{k, \varepsilon}$ (recall b) stated before Proposition 3.3) that $\left|R_{s_{s_{i-1}+1}}\left(n_{k_{i-1}}, n_{k_{i}-1} ; E\right)\right|<\varepsilon\left\langle n_{k_{i-1}}-n_{k_{i}-1}\right\rangle^{-7}$, and it is elementary to establish Relation (3.31) below. So we consider only the case $s_{i-1}+1>k$. We now have that $l_{s_{i-1}+1}>C / \varepsilon$ (recall (3.18) and the definition of $\mathcal{M}_{k, \varepsilon}$ ). We will also use the estimate (3.28), with the previous convention of droping the last term and using a term $\left\langle n_{k_{i-1}}-b_{s_{i-1}}\right\rangle^{-\gamma / 4}$ arising from $j\left(n_{k_{i-2}}-n_{k_{i-1}}\right)$. Using these facts, one shows that

$$
\sum_{\substack{n_{k_{i-1}}, n_{k_{i}-1} \in I_{s_{i-1}+1} \\ \mid n_{k_{i}-1}-a_{s_{i-1}+1}>3 l_{s_{i-1}+1 / 4}}}\left|R_{I_{s_{i-1}+1}}\left(n_{k_{i-1}}, n_{k_{i}-1} ; E\right)\right| \cdot \mid j\left(n_{k_{i}-1}-n_{k_{i}} \mid \leq C \varepsilon\left\langle s_{i-1}\right\rangle^{-1-\delta}\left\langle d\left(s_{i-1}, s_{i}\right)\right\rangle^{-\gamma / 4},\right.
$$

by distingushing the cases $\left|n_{k_{i-1}}-n_{k_{i}-1}\right|>l_{s_{i-1}+1} / 2\left(\leq l_{s_{i-1}+1} / 2\right)$. In the first one uses $(3.18)$ and in the second case that $n_{k_{i-1}}-b_{s_{i-1}}>l_{s_{i-1}+1} / 4>C / \varepsilon$. 
To handle the second term in (3.30) one argues similarly as in the Case 2. Thus we again arrive at the estimate

$$
\sum_{n_{k_{i-1}}, n_{k_{i}-1} \in I_{s_{i-1}+1}}\left|R_{M_{s_{i-1}}}\left(n_{k_{i-1}}, n_{k_{i}-1} ; E\right)\right| \cdot\left|j\left(n_{k_{i}-1}-n_{k_{i}}\right)\right| \leq C \varepsilon\left\langle s_{i-1}\right\rangle^{-1-\delta}\left\langle d\left(s_{i-1}, s_{i}\right)\right\rangle^{-\gamma / 4} .
$$

Cases 4- 6 . In each of these cases, the path jumps over the long block $I_{s_{i-1}+1}$. Arguing as in Case 1, one derives estimates analogous to (3.24) and (3.25).

We add that a similar argument shows that

$$
\sum_{t \in M_{0}}\left|R_{M_{0}}(0, t ; E) j\left(t-n_{1}\right)\right| \leq C \varepsilon\left\langle d\left(0, s_{1}\right)\right\rangle^{-\gamma / 4}
$$

for a suitable constant $C$. Since the first parameter is fixed to be zero, here we do not need a contribution from $j$ on the left-hand side to compensate for the sum with respect to this parameter.

We emphasize that for all the other initial arrangments of the main blocks $M_{s_{i-2}}, M_{s_{i-1}}, M_{s_{i}}$ and $M_{s_{i+1}}$, a similar argument leads to the estimates analogous to (3.25), (3.29) and (3.31). We also remark that in our analysis the blocks $M_{s_{i-2}}$ and $M_{s_{i+1}}$ played a minor role - they contributed only in a sense that a certain part of the estimate is carried over to be used in the estimation of the next term.

We now go back to the formal expansion (3.21). It follows from the above considerations that

$$
\begin{gathered}
\delta_{0 m} /|v(0)-E|+\sum_{\tau_{b} \in \mathcal{B}}\left|R_{M_{0}}\left(0, n_{k_{1}-1} ; E\right)\right|\left|j\left(n_{k_{1}-1}-n_{k_{1}}\right)\right|\left|R_{M_{s_{1}}}\left(n_{k_{1}}, n_{k_{2}-1} ; E\right)\right| \ldots \\
\ldots\left|R_{M_{s_{l-1}}}\left(n_{k_{l-1}}, n_{k_{l}-1} ; E\right)\right|\left|j\left(n_{k_{l}-1}-n_{k_{l}}\right)\right|\left|R_{M_{n_{0}}}\left(n_{k_{l}}, m ; E\right)\right| \\
\leq \delta_{0 m} /|v(0)-E|+\sum_{j \geq 1}\left(C \varepsilon \sum_{k>0} \frac{1}{k^{1+\delta}}\right)^{j} G(m) .
\end{gathered}
$$

Here $C$ is a constant which depends only on $\delta_{0}$ in $(3.14)$, and

$$
G(m) \equiv \max _{i} \sum_{t \in M_{n_{0}}}\left\langle x_{n_{0}}^{(i)}-t\right\rangle^{-\gamma / 4}\left|R_{M_{n_{0}}}(t, m ; E)\right| .
$$

The contribution $\left\langle x_{n_{0}}^{(i)}-t\right\rangle^{-\gamma / 4}$ is the remaining part of the estimate analogous (for example) to (3.28) used in the estimation of (3.21) in the case $s_{i-1}=s_{l-1}$ (recall the conventions introduced in the derivation of (3.25), (3.29), (3.31) and the form of the expansion (3.21)). Thus, if $\varepsilon$ is chosen so that

$$
C \varepsilon \zeta(1+\delta)<\frac{1}{3}
$$

we get that for $k>k(\varepsilon)$, a.e. $E \in M_{k, \varepsilon}, \forall \ell$ and $\forall m \in J_{\ell}$, the formal series (3.21) converges absolutely. Here and in the sequel, $\zeta$ is the usual Riemann function. 
We remark that in the estimation (3.32) we have not used the contributions arising from $d\left(s_{i-1}, s_{i}\right)$, and it follows from analysis that all the conclusions of the previous paragraph hold for any $\gamma>6$. The contributions arising from $d\left(s_{i-1}, s_{i}\right)$ are however essential to estimate the sum (3.22) uniformly in $\ell$. To that end we need an improvement of the estimate (3.32).

We split the set of bonds $\mathcal{B}$ as $\mathcal{B}=\mathcal{B}_{1} \cup \mathcal{B}_{2}$ where $\mathcal{B}_{1}\left(\mathcal{B}_{2}\right)$ consists of bonds associated to the paths whose length is $<n_{0} / 2\left(\geq n_{0} / 2\right)$. Accordingly, we decompose $\mathcal{R}_{\ell}(0, m ; E)$ as

$$
\mathcal{R}_{\ell}(0, m ; E)=A_{1}(0, m ; E)+A_{2}(0, m ; E) .
$$

To simplify the notation, we will assume that $n_{0}>0$ is even, $n_{0}=2 n_{0}^{\prime}$. A similar argument applies if $n_{0}$ is odd or if $n_{0}=0$. To estimate $A_{2}$ we again do not need the contributions arising from $d\left(s_{i-1}, s_{i}\right)$. It $\varepsilon$ is chosen so that (3.33) holds, we get that for $E \in \mathcal{M}_{k, \varepsilon}$,

$$
\left|A_{2}(0, m ; E)\right| \leq \sum_{j \geq n_{0}^{\prime}}[C \varepsilon \zeta(1+\delta)]^{j} G(m) \leq 2[C \varepsilon \zeta(1+\delta)]^{n_{0} / 2} G(m),
$$

We now proceed to estimate $A_{1}$. Here, we have to make use of the contributions arising from $d\left(s_{i-1}, s_{i}\right)$. Let us split $\mathcal{B}_{1}$ into sets of disjoint bonds such that the associated paths have lengths $1,2, \ldots, n_{0}^{\prime}-1$. Accordingly, $A_{1}$ splits as

$$
A_{1}=\sum_{l=1}^{n_{0}^{\prime}-1} A^{(l)} .
$$

To estimate $A^{(l)}(0, m ; E)$, we note that if the path has the length $l$ and $n_{0}>2 l$, then at least one of $d\left(s_{i-1}, s_{i}\right)$ satisfies $d\left(s_{i-1}, s_{i}\right) \geq 2^{n_{0}-2 l}$. This leads to the estimate

$$
\left|A^{(l)}(0, m ; E)\right| \leq 2^{-\gamma n_{0} / 4}\left(C \varepsilon \zeta(1+\delta) 2^{\gamma / 2}\right)^{l}
$$

If we choose $\varepsilon$ such that $C \varepsilon \zeta(1+\delta) 2^{\gamma / 2}<1 / 3$, we arrive at the estimate

$$
\left|A_{1}(0, m ; E)\right| \leq 2 \cdot 2^{-\gamma n_{0} / 4} G(m)
$$

Thus, we summarize: if $\varepsilon$ is sufficiently small, and $k>k(\varepsilon)$, then for all $E \in \mathcal{M}_{k, \varepsilon}$ we have a sharper estimate then (3.32):

$$
\left|\mathcal{R}_{\ell}(0, m ; E)\right|^{2} \leq 4\left((C \varepsilon \zeta(1+\delta))^{n_{0} / 2}+2^{-\gamma n_{0} / 4}\right)^{2} G(m)^{2}
$$

We proceed to estimate $G(m)^{2}$. Let $L_{n}=\left|M_{n}\right|+1$. Then

$$
G(m)^{2} \leq L_{n_{0}} \max _{i} \sum_{t \in M_{n_{0}}}\left\langle x_{n_{0}}^{(i)}-t\right\rangle^{-4(1+\delta)}\left|R_{M_{n_{0}}}(t, m ; E)\right|^{2}
$$


This inequality can be rewritten as

$$
G(m)^{2} \leq L_{n_{0}} \sum_{p}\langle p\rangle^{-4(1+\delta)} \max _{i}\left|R_{M_{n_{0}}}\left(x_{n_{0}}^{(i)}+p, m ; E\right)\right|^{2}
$$

It now follows from Proposition 3.2 that

$$
\sum_{m \in M_{n_{0}}} G(m)^{2} \leq L_{n_{0}}^{2} \zeta(2(1+\delta))\left\langle n_{0}\right\rangle^{2(1+\delta)} \leq C 2^{2 n_{0}}\left\langle n_{0}\right\rangle^{2(1+\delta)}
$$

We have absorbed $N$ from (3.11) into the constant $C$. Choosing $\varepsilon$ in (3.34) so that $C \varepsilon \zeta(1+\delta)<$ $1 / 2^{5}$, it follows easily from (3.34) and (3.35) that for $E \in \mathcal{M}_{k, \varepsilon}$,

$$
\sum_{m \in M_{n_{0}}}\left|\mathcal{R}_{\ell}(0, m ; E)\right|^{2} \leq C 2^{-n_{0} / 2}\left\langle n_{0}\right\rangle^{2(1+\delta)}
$$

Thus, we conclude: if $\varepsilon_{0}$ is chosen sufficiently small, $k>k\left(\varepsilon_{0}\right)$, and $E \in \mathcal{M}_{k, \varepsilon_{0}}$, then

$$
\begin{aligned}
\sum_{m \in J_{\ell}}\left|\mathcal{R}_{\ell}(0, m ; E)\right|^{2} & \leq \sum_{n,|n| \leq \ell} \sum_{m \in M_{n}}|\mathcal{R}(0, m ; E)|^{2} \\
& \leq C \sum_{n} 2^{-|n| / 2}\langle n\rangle^{2(1+\delta)}
\end{aligned}
$$

where the constant $C$ does not depend on $\ell$. This yields (3.22). The reader can easily convince himself that none of the constants in above arguments depends on the particular choice $i=0$ and therefore that Proposition 3.3 holds, with the exactly same argument, for each $i \in \cup_{s=-k}^{k} M_{s}$.

Finally, we remark that if $j(n)$ decays faster then any polynomial then $\gamma$ can be taken arbitrarily large. We leave it as an exercise for the reader to show that in that case for a.e. $E \in \mathcal{I}$ and any $m$ we have that

$$
\sup _{0<\zeta<1}\left|\left(\delta_{0},(h-E-i \zeta)^{-1} \delta_{m}\right)\right| \leq C_{E, k}\langle m\rangle^{-k}
$$

for any $k>0$. This estimate combined with Simon-Wolff theorem [SW] will yield the decay of eigenfunctions described in Remark 2 after Theorem 1.1.

\section{Periodic potentials and gaps}

In this section we study the operators $h_{\varepsilon, p}=h_{0}+v_{\varepsilon, p}$, where $v_{\varepsilon, p}$ is a periodic potential of the form

$$
v_{\varepsilon, p}(n)= \begin{cases}\varepsilon & \text { if } n \equiv 0 \bmod p, \\ 0 & \text { if } n \neq 0 \bmod p .\end{cases}
$$

Here $p$ is a positive integer and $\varepsilon>0$ a positive parameter. The operator $h_{0}$ is given by (1.2), and in the Fourier representation, it acts as the operator of multiplication by the function $\hat{j}(\varphi)$. 
In this section we make the following assumption on $\hat{j}(\varphi)$ :

(H) $\hat{j}(\varphi)$ is a continuous real even function, strictly monotone and twice continuosly differentiable on $(0, \pi)$.

Remark. Note that conditions of Theorem 1.1 imply $(\mathbf{H})$. This hypothesis allows for some mild singularity of $\hat{j}^{\prime}$ at $\varphi=0$. For example, it is satisfied if $j(n)=|n|^{-\gamma}$ and $\gamma>1$.

Notation. In the sequel we will use the shorthand $e_{k, p}=\hat{j}(k \pi / p)$.

The principal result of this section is

Theorem 4.1 Assume that $(\mathbf{H})$ holds and let $\left(\theta_{1}, \theta_{2}\right) \subset \sigma\left(h_{0}\right)$. Then there exists $\varepsilon_{0}>0$ and $p_{0}>0$, which depend only on $\theta_{1}$ and $\theta_{2}$, such that for $0<\varepsilon<\varepsilon_{0}, p>p_{0}$, and $e_{k, p} \in\left(\theta_{1}, \theta_{2}\right)$,

$$
\sigma\left(h_{\varepsilon, p}\right) \cap\left(e_{k, p}, e_{k, p}+\delta_{\varepsilon, k, p}\right)=\emptyset
$$

for some $\delta_{\varepsilon, k, p}>0$.

Remark. If $\hat{j}(\varphi)=2 \cos \varphi$, a similar result was proven in [KKS].

Notation. In the sequel, whenever there is no danger of confusion, we write $h$ for $h_{\varepsilon, p}$ and $R(z)$ for $\left(h_{\varepsilon, p}-z\right)^{-1}$.

The rest of this section is devoted to the proof of Theorem 4.1.

Our argument is based on

Proposition 4.2 Let $\mathcal{I}$ be an open interval. If for all $E \in \mathcal{I}$ and $n \in \mathbf{Z}$,

$$
\lim _{\zeta \downarrow 0} \sum_{m}|R(m, n ; E+i \zeta)|^{2}<\infty
$$

then $\sigma(h) \cap \mathcal{I}=\emptyset$.

Proof: Fix $n \in \mathbf{Z}$ and let $\mu_{n}$ be the spectral measure associated to the vector $\delta_{n}$. We denote by $F(z)$ the Borel transform of $\mu_{n}$, i.e.

$$
F(z) \equiv \int \frac{d \mu_{n}(\lambda)}{\lambda-z}=\left(\delta_{n},(h-z)^{-1} \delta_{n}\right)
$$

It follows from the theorems of Fatou and de Vallée Poussin (see e.g. Chapter 1 in [S] for detailed discussion) that

$$
\operatorname{supp} \mu_{n} \subset \overline{\left\{E: \limsup _{\zeta \downarrow 0} \operatorname{Im} F(E+i \zeta)>0\right\}} .
$$

Since

$$
\operatorname{Im} F(E+i \zeta)=\zeta \sum_{m}|R(m, n ; E+i \zeta)|^{2},
$$


it follows from (4.37) that $\operatorname{supp} \mu_{n} \cap \mathcal{I}=\emptyset$. Finally, since

$$
\sigma(h)=\overline{\bigcup_{n \in \mathbf{Z}} \operatorname{supp} \mu_{n}}
$$

we derive that $\sigma(h) \cap \mathcal{I}=\emptyset$.

We will prove Theorem 4.1 by showing that (4.37) holds for each $0 \leq n \leq p-1$ and $E \in\left(e_{k, p}, e_{k, p}+\delta_{\varepsilon, k, p}\right)$. Since $R(n+p, m+p ; z)=R(n, m ; z)$, this suffices. Let

$$
G^{(n)}(\varphi ; z)=\frac{1}{\sqrt{2 \pi}} \sum_{m} e^{i m \varphi} R(m, n ; z)
$$

Since

$$
\int_{0}^{2 \pi}\left|G^{(n)}(\varphi ; E+i \zeta)\right|^{2} d \varphi=\sum_{m}|R(m, n ; E+i \zeta)|^{2}
$$

to prove Theorem 4.1 it suffices to show that for each $e_{k, p} \in\left(\theta_{1}, \theta_{2}\right)$, there exists $\delta_{\varepsilon, k, p}>0$ such that for all $E \in\left(e_{k, p}, e_{k, p}+\delta_{\varepsilon, k, p}\right)$ and $0 \leq n \leq p-1$, the limit

$$
\lim _{\zeta \downarrow 0} G^{(n)}(\varphi, E+i \zeta)
$$

exists uniformly in $\varphi$. To establish this fact, we proceed to compute $G^{(n)}(\varphi ; z)$.

The Fourier transform of the resolvent equation

$$
\left(h_{0}+\varepsilon v_{p}(m)\right) R(m, n ; z)=z R(m, n, z)+\delta_{n},
$$

is

$$
\hat{j}(\varphi) G^{(n)}(\varphi ; z)+\varepsilon S^{(n)}(\varphi ; z)=z G^{(n)}(\varphi, z)+e^{i n \varphi} / \sqrt{2} \pi,
$$

where

$$
S^{(n)}(\varphi ; z)=\frac{1}{\sqrt{2 \pi}} \sum_{m} e^{i m p \varphi} R(m p, n ; z)
$$

Notation. In the sequel, $n$ is a fixed integer, and we will drop superscripts $(n)$ whenever there is no danger of confusion.

Let

$$
\begin{aligned}
G_{l}(\varphi ; z) & \equiv G(\varphi+2 \pi l / p ; z) \\
\hat{j}_{l}(\varphi) & \equiv \hat{j}(\varphi+2 \pi l / p) \\
\chi_{l}(\varphi) & \equiv e^{i n(\varphi+2 \pi l / p)} / \sqrt{2 \pi}
\end{aligned}
$$

We write $\chi_{0}=\chi, G_{0}=G$. Since $S(\varphi+2 \pi l / p ; z)=S(\varphi ; z)$, translating the argument in Equation (4.39) by $2 \pi l / p$, we get

$$
\hat{j}_{l} G_{l}+\varepsilon S=z G_{l}+\chi_{l} .
$$


Thus, for $0 \leq l \leq p-1$,

$$
G_{l}=\frac{\chi_{l}-\varepsilon S}{\hat{j}_{l}-z}
$$

Since

$$
\begin{aligned}
\sum_{l=0}^{p-1} G_{l} & =\frac{1}{\sqrt{2 \pi}} \sum_{l=0}^{p-1} \sum_{m} e^{i m(\varphi+2 \pi l / p)} R(m, n ; z) \\
& =\frac{1}{\sqrt{2 \pi}} \sum_{m} e^{i m \varphi} R(m, n ; z) \sum_{l=0}^{p-1} e^{l(2 \pi i m / p)} \\
& =p S(\varphi ; z)
\end{aligned}
$$

adding together Equations (4.40) and solving for $S$ we get

$$
S=\left(\frac{1}{p} \sum_{l=0}^{p-1} \frac{\chi_{l}}{\hat{j}_{l}-z}\right)\left(1+\frac{\varepsilon}{p} \sum_{l=0}^{p-1} \frac{1}{\hat{j}_{l}-z}\right)^{-1} .
$$

It now follows from Equation (4.40) that

$$
G=\frac{\chi+K_{1}}{(\hat{j}-z)\left(1+K_{2}\right)}
$$

where

$$
K_{1}=\frac{\varepsilon}{p} \sum_{l=1}^{p-1} \frac{\chi-\chi_{l}}{\hat{j}_{l}-z}, \quad K_{2}=\frac{\varepsilon}{p} \sum_{l=0}^{p-1} \frac{1}{\hat{j}_{l}-z} .
$$

Note that $K_{2}$ is a periodic function with a period $2 \pi / p$.

This explicit expression for $G$ will play a central role in the sequel. The principal technical ingredient in our proof is

Proposition 4.3 Assume that the conditions of Theorem 4.1 are satisfied and let $e_{k, p} \in\left(\theta_{1}, \theta_{2}\right)$. Then there exists $\varepsilon_{0}>0$ and $p_{0}>0$, which depend only on $\theta_{1}, \theta_{2}$, such that for $0<\varepsilon<\varepsilon_{0}$ and $p>p_{0}$,

$$
\inf _{\varphi}\left|1+K_{2}(\varphi, E)\right| \geq 1 / 2
$$

for all $E \in\left(e_{k, p}, e_{k, p}+\delta_{\varepsilon, k, p}\right)$ and some $\delta_{\varepsilon, k, p}>0$.

Let us show how this proposition yields Relation (4.38) and Theorem 4.1.

Let $E \in\left(e_{k, p}, e_{k, p}+\delta_{\varepsilon, k, p}\right)$ and let $\varphi^{+}$and $\varphi^{-}$be the unique solutions of equation $\hat{j}(\varphi)=E$ which belong to intervals $(0, \pi)$ and $(-\pi, 0)$ respectively. Let

$$
\varphi_{l}^{ \pm}=\varphi^{ \pm}+2 l \pi / p, \quad 0 \leq l \leq p-1 .
$$

Then it follows from (4.41)-(4.43) that the limit

$$
\lim _{\zeta \downarrow 0} G(\varphi ; E+i \zeta)
$$


exists uniformly in $\varphi$ on compact intervals which do not contain the points $\varphi_{l}^{ \pm}$. If $\delta_{\varepsilon, k, p}$ is chosen so that, $\forall j, e_{j, p} \notin\left(e_{k, p}, e_{k, p}+\delta_{\varepsilon, k, p}\right)$, then $\varphi^{+}-\varphi^{-}$is not an integer multiple of $2 \pi / p$. Thus, if $l \neq 0$, only the terms with the index $p-l$ in the sums (4.42) do become singular in a small neighborhod of $\varphi_{l}^{ \pm}$, and these two terms cancel each other in expression (4.41). More precisely, if $0<l_{0} \leq p-1$ is fixed, we can rewrite (4.41) in a small neighborhood of the singular points $\varphi_{l_{0}}^{ \pm}$as $G=R_{1} / R_{2}$, where

$$
\begin{aligned}
& R_{1}=\chi\left(\hat{j}_{p-l_{0}}-z\right)+\frac{\varepsilon}{p}\left(\left(\chi-\chi_{p-l_{0}}\right)+\sum_{l=1, l \neq p-l_{0}}^{p-1} \frac{\left(\hat{j}_{p-l_{0}}-z\right)\left(\chi-\chi_{l}\right)}{\hat{j}_{l}-z}\right) \\
& R_{2}=(\hat{j}-z)\left(\left(\hat{j}_{p-l_{0}}-z\right)+\frac{\varepsilon}{p}\left(1+\sum_{l=0, l \neq p-l_{0}}^{p-1} \frac{\hat{j}_{p-l_{0}}-z}{\hat{j}_{l}-z}\right)\right)
\end{aligned}
$$

It follows that the limit (4.44) exist uniformly in $\varphi$ in a small neighborhood of any point $\varphi_{l}^{ \pm}$, $l \neq 0$. It remains to consider the case $l=0$. We rewrite $G$ in small neighborhoods of the points $\varphi^{ \pm}$as $G=\left(\chi+K_{1}\right) / R_{3}$, where

$$
R_{3}=(\hat{j}-z)+\frac{\varepsilon}{p}+\frac{\varepsilon}{p} \sum_{l=1}^{p-1} \frac{\hat{j}-z}{\hat{j}_{l}-z} .
$$

It follows that the limit (4.44) exists uniformly in $\varphi$ in small neigborhoods of points $\varphi^{ \pm}$, and therefore for all $\varphi$.

It remains to prove Proposition 4.2. We will need

Lemma 4.4 Let $f:[-a, a] \mapsto \mathbf{R}$ be a strictly monotone, twice continuosly differentiable function. For $0<|x|<h<a / 2$ we define a function

$$
F(x, h)=\sum_{\substack{k \\ x+k h \in[-a, a]}} \frac{h}{f(x+k h)-f(0)}
$$

Let $\alpha=|x| / h$. Then

$$
F(x, h)=\frac{\operatorname{sign}(x)}{f^{\prime}(0)}\left[\frac{1}{\alpha}-\frac{1}{1-\alpha}\right]+r(x, h)
$$

where

$$
|r(x, h)| \leq \frac{2}{\left|f^{\prime}(0)\right|}\left(1+a \frac{\max \left|f^{\prime \prime}(x)\right|}{\min \left|f^{\prime}(x)\right|}\right) .
$$

Proof: We will discuss only the case $x>0$, one argues similarly if $x<0$. Write $F=F_{1}+F_{2}$, where

$$
F_{1}(x, h)=\sum_{\substack{k \\ x+k h \in[0, a]}} \frac{h}{f(x+k h)-f(0)}, \quad F_{2}(x, h)=\sum_{\substack{k \\ x+k h \in[-a, 0]}} \frac{h}{f(x+k h)-f(0)} .
$$


We deal first with $F_{1}$. Let $n_{x}=\max \{k: x+k h \in[0, a]\}$. Note that if $n$ is the integer such that $(n+1) h \leq a<(n+2) h$, then $n \leq n_{x} \leq n+1$. It follows from Taylors formula that

$$
\left|\frac{1}{f(x+k h)-f(0)}-\frac{1}{f^{\prime}(0)(x+k h)}\right| \leq \frac{1}{2\left|f^{\prime}(0)\right|} \frac{\max _{x \in[0, a]}\left|f^{\prime \prime}(x)\right|}{\min _{x \in[0, a]}\left|f^{\prime}(x)\right|}
$$

Since $\left(n_{x}+1\right) h<3 a / 2$, we have that

$$
F_{1}(x, h)=\frac{1}{f^{\prime}(0) \alpha}+\sum_{k=1}^{n_{x}} \frac{1}{f^{\prime}(0)(k+\alpha)}+r_{1}(x, h)
$$

where

$$
\left|r_{1}(x, h)\right|<\frac{a}{\left|f^{\prime}(0)\right|} \frac{\max _{x \in[0, a]}\left|f^{\prime \prime}(x)\right|}{\min _{x \in[0, a]}\left|f^{\prime}(x)\right|} .
$$

If $g(x)=f(-x)$, the same argument yields

$$
F_{2}(x, h)=h \sum_{k=0}^{n_{h-x}} \frac{1}{g(h-x+k h)-g(0)}=\frac{1}{g^{\prime}(0)(1-\alpha)}+\sum_{k=1}^{n_{h-x}} \frac{1}{g^{\prime}(0)(k+1-\alpha)}+r_{2}(x, h),
$$

where $r_{2}(x, h)$ satisfies the estimate analogous to (4.46). Since $g^{\prime}(0)=-f^{\prime}(0)$, we get that

$$
\begin{aligned}
F(x, h)= & \frac{1}{f^{\prime}(0)}\left[\frac{1}{\alpha}-\frac{1}{1-\alpha}\right]+\frac{1}{f^{\prime}(0)} \sum_{k=1}^{n} \frac{1-2 \alpha}{(k+\alpha)(k+1-\alpha)} \\
& +\frac{1}{f^{\prime}(0)}\left[\frac{\delta_{n+1, n_{x}}}{n+1+\alpha}-\frac{\delta_{n+1, n_{h-x}}}{n+2-\alpha}\right]+r_{1}(x, h)+r_{2}(x, h) .
\end{aligned}
$$

Since $0<\alpha<1$, the result follows.

We now finish the proof of Proposition 4.2. For definitness, we assume that $\hat{j}^{\prime}(\varphi)<0$ on $(0, \pi)$. Let $\varphi_{1}, \varphi_{2} \in(0, \pi)$ be such that $\hat{j}\left(\varphi_{i}\right)=\theta_{i}$. Choose $p_{0}$ such that $4 \pi / p_{0}<\min \left\{\varphi_{1}, \pi-\varphi_{2}\right\}$. Let $p>p_{0}$ and $e_{k, p} \in\left(\theta_{1}, \theta_{2}\right)$ be given. Let $\delta>0$ be a small number. For each $E \in\left(e_{k, p}, e_{k, p}+\delta\right)$ we denote by $\varphi_{E}^{ \pm}$respectively the positive and negative solution of the equation $\hat{j}(\varphi)=E$. We will study $K_{2}(\varphi ; E)$ for $\varphi \in\left(\varphi_{E}^{+}, \varphi_{E}^{+}+2 \pi / p\right)$. We can choose $\delta$ sufficently small so that for some $b>4 \pi / p$, the intervals $\left[\varphi_{E}^{ \pm}-b, \varphi_{E}^{ \pm}+b\right] \subset(-\pi, \pi)$ for all $E \in\left(e_{k, p}, e_{k, p}+\delta\right)$. If $\varphi \in\left(\varphi_{E}^{+}, \varphi_{E}^{+}+2 \pi / p\right)$, we split $K_{2}(\varphi, E)$ into three terms,

$$
K_{2}(\varphi ; E)=K_{2}^{+}(\varphi ; E)+K_{2}^{-}(\varphi ; E)+\tilde{K}_{2}(\varphi, E),
$$

where

$$
\begin{aligned}
& K_{2}^{+}(\varphi ; E)=\sum_{\substack{l \\
\varphi+2 \pi l / p \in\left[\varphi^{+}-b, \varphi^{+}+b\right]}} \frac{\varepsilon}{p} \frac{1}{\hat{j}_{l}(\varphi)-E}, \\
& K_{2}^{-}(\varphi ; E)=\sum_{\substack{l \\
\varphi+2 \pi l / p \in\left[\varphi^{-}-b, \varphi^{-}+b\right]}} \frac{\varepsilon}{p} \frac{1}{\hat{j}_{l}(\varphi)-E},
\end{aligned}
$$




$$
\tilde{K}_{2}(\varphi ; E)=\sum_{\varphi+2 \pi l / p \notin\left[\varphi^{l}-b, \varphi^{ \pm}+b\right]} \frac{\varepsilon}{p} \frac{1}{\hat{j}_{l}(\varphi)-E} .
$$

From this definition it follows that for all $\varphi \in\left(\varphi^{+}, \varphi^{+}+2 \pi / p\right)$,

$$
\left|\tilde{K}_{2}(\varphi, E)\right|<C \varepsilon \text {, }
$$

where the constant $C$ depends only on $b$ and the function $\hat{j}$. To analyze the terms $K_{2}^{+}$and $K_{2}^{-}$, we will use Lemma 4.3. It is apparent that, after translation, the sum which constitute $K_{2}^{+}$and is of the form (4.45), with $h=2 \pi / p$ and

$$
\alpha=\frac{\varphi-\varphi_{E}^{+}}{h} .
$$

It then follows from Lemma 4.3 that

$$
K_{2}^{+}(\varphi ; E)=\frac{\varepsilon}{2 \pi \hat{j}^{\prime}\left(\varphi_{E}^{+}\right)}\left[\frac{1}{\alpha}-\frac{1}{1-\alpha}\right]+\varepsilon r_{E}^{+}(\varphi ; E) .
$$

The error term $r_{E}^{+}$is uniformly bounded and the bound depends only on $b$ and $\hat{j}$. Note that

$$
\varphi_{E}^{+}-\varphi_{E}^{-}=\frac{2 k \pi}{p}+\frac{2 \pi \gamma_{E}}{p},
$$

where $\gamma_{E}<0$ if $E>e_{k, p}$. Furhermore, $\gamma_{E}$ is a continuous function of $E$ in a neighborhood of $e_{k, p}$ and $\gamma_{E} \rightarrow 0$ as $E \rightarrow e_{k, p}$. Thus, we can choose $\delta$ such that $\left|\gamma_{E}\right|<1 / 2$ for $E \in\left(e_{k, p}, e_{k, p}+\delta\right)$. One now easily shows that, after translation, the sum which consitute $K_{2}^{-}$is of the form (4.45) with $h=2 \pi / p$ and

$$
\alpha^{\prime}=\frac{\left|\varphi-\varphi_{E}^{+}-2 k \pi / p\right|}{h}=\left|\alpha+\gamma_{E}\right| .
$$

It then follows from Lemma 4.3 that

$$
K_{2}^{-}(\varphi ; E)=\frac{\varepsilon}{2 \pi \hat{j}^{\prime}\left(\varphi_{E}^{-}\right)}\left[\frac{1}{\alpha+\gamma_{E}}-\frac{\operatorname{sign}\left(\alpha+\gamma_{E}\right)}{1-\left|\alpha+\gamma_{E}\right|}\right]+\varepsilon r_{E}^{-}(\varphi ; E),
$$

where again $r_{E}^{-}(\varphi ; E)$ is uniformly bounded and the bound depends only on $b$ and $\hat{j}$. Thus, we conclude that for $\varphi \in\left(\varphi_{E}^{+}, \varphi_{E}^{+}+2 \pi / p\right)$,

$$
K_{2}=\frac{\varepsilon}{2 \pi \hat{j}^{\prime}\left(\varphi_{E}^{+}\right)} \frac{\gamma_{E}}{\alpha\left(\alpha+\gamma_{E}\right)}+\varepsilon\left(R_{1}+R_{2}\right) x+\tilde{K}_{2},
$$

where

$$
R_{1}=\frac{1}{\hat{j}^{\prime}\left(\varphi_{E}^{+}\right)}\left[\frac{1}{1-\alpha}-\frac{\operatorname{sign}\left(\alpha+\gamma_{E}\right)}{1-\left|\alpha+\gamma_{E}\right|}\right], \quad R_{2}(\varphi ; E)=r_{E}^{-}+r_{E}^{+} .
$$

Furthermore, $R_{1}$ is always positive while $R_{2}$ is bounded. Finally, it remains to analyze the function

$$
\frac{\varepsilon}{2 \pi \hat{j}^{\prime}\left(\varphi^{+}\right)} \frac{\gamma_{E}}{\alpha\left(\alpha+\gamma_{E}\right)}
$$


for $0<\alpha<1$. This function is negative on the interval $\left(0,\left|\gamma_{E}\right|\right)$, and positive on the interval $\left(\left|\gamma_{E}\right|, 1\right)$, with vertical assymptotes at 0 and $\left|\gamma_{E}\right|$. The maximal value of the function on the interval $\left(0,\left|\gamma_{E}\right|\right)$ is

$$
\frac{2 \varepsilon}{\pi \hat{j}^{\prime}\left(\varphi_{E}^{+}\right)\left|\gamma_{E}\right|} .
$$

Choose now $\varepsilon_{0}$ such that $\tilde{K}_{2}<1 / 4$ (recall (4.47)), $\varepsilon_{0}\left|R_{2}\right|<1 / 4$ and that $\varepsilon_{0} R_{1}<1 / 4$ for $\alpha \in(0,1 / 2)$. Note that $\varepsilon_{0}$ depends only on $b$ and on the function $\hat{j}$. Since $\gamma_{E} \rightarrow 0$ as $E \rightarrow e_{k, p}$, we can find $\delta$ such that for all $E \in\left(e_{k, p}, e_{k, p}+\delta\right)$, and for $\alpha \in\left(0,\left|\gamma_{E}\right|\right)$ the function (4.48) is less then -3 . Thus, we summarize: there exists $\delta_{\varepsilon, k, p}>0$ such that for all $E \in\left(e_{k, p}, e_{k, p}+\delta_{\varepsilon, k, p}\right)$ and $\varphi \in\left(\varphi_{E}^{+}, \varphi_{E}^{+}+2 \pi / p\right)$,

$$
\left|1+K_{2}(\varphi ; E)\right| \geq 1 / 2
$$

The Proposition 4.2 follows.

\section{Dirichlet decoupling}

Let $v$ be a periodic potential with the period $p$, and let $h_{0}^{L}$ be the operator $h_{0}$ with Dirichlet boundary condition at $\pm 2 p L$,

$$
h_{0}^{L}=h_{0}-\sum_{|k| \leq 2 p L,\left|k^{\prime}\right|>2 p L} j\left(k^{\prime}-k\right)\left(\delta_{k}, \cdot\right) \delta_{k^{\prime}}+j\left(k-k^{\prime}\right)\left(\delta_{k^{\prime}}, \cdot\right) \delta_{k} .
$$

Note that the operator $h_{0}^{L}$, restricted to $l^{2}([-2 p L, 2 p L])$, coincides with operator $h_{0}^{D}$ defined by (2.8) if $I=[-2 p L, 2 p L]$. We set $h=h_{0}+v, h^{L}=h_{0}^{L}+v$. Let $(a, b)$ be an interval such that $0 \notin(a, b)$ and

$$
\sigma(h) \cap(a, b)=\emptyset .
$$

For any $L, h^{L}-h$ is a trace class operator, and the spectrum of $h^{L}$ within $(a, b)$ consists of (possibly empty) discrete set of eigenvalues of finite multiplicity which can accumulate only at $a$ and $b$. We denote this set of eigenvalues by $\mathcal{S}_{L}=\left\{E_{i}(L)\right\}$. In the next section we will need some control over the set $\mathcal{S}_{L}$ as $L \uparrow \infty$ to verify the conditions of Theorem 3.1 for random Hamiltonians (1.1). The following technical result will suffice.

Proposition 5.1 Assume that $\gamma>2$ and let $\epsilon>0$ and $\delta>0$ be given small numbers. Then there exists finitely many points $r_{1}, \ldots, r_{k_{\epsilon, \delta}}$ in $(a+\epsilon, b-\epsilon)$ and a positive number $L_{\epsilon, \delta}$, such that for $L>L_{\epsilon, \delta}$,

$$
\mathcal{S}_{L} \cap(a+\epsilon, b-\epsilon) \subset \cup_{l=1}^{k_{\epsilon, \delta}}\left[r_{l}-\delta, r_{l}+\delta\right] .
$$

The points $r_{l}$ and the numbers $L_{\epsilon, \delta}$ and $k_{\epsilon, \delta}$ depend only on $\epsilon$ and $\delta$. Furthermore, $\sup _{\delta>0} k_{\epsilon, \delta} \leq$ $k_{\epsilon}<\infty$, where $k_{\epsilon}$ depends only on $\epsilon$. 
This section is devoted to the proof of this proposition.

In our argument we will make use of the following three simple facts.

Lemma 5.2 Let $A: l^{2}(\mathbf{Z}) \mapsto l^{2}(\mathbf{Z})$ be a selfadjoint operator and let $A_{i j}=\left(\delta_{i}, A \delta_{j}\right)$. Let

$$
M_{1}=\sup _{j} \sum_{i}\left|A_{i j}\right|, \quad M_{2}=\sup _{i} \sum_{j}\left|A_{i j}\right|
$$

Then $\|A\| \leq \max \left\{M_{1}, M_{2}\right\}$.

For the proof, see [Ka], Section 1.4.3, or Lemma 7.1 in [SS].

Lemma 5.3 Let $A$ and $B$ be bounded selfadjoint operators. Then

$$
\sigma(A+B) \subset \sigma(A)+[-\|B\|,\|B\|]
$$

Proof: Assume that $E \notin \sigma(A)+[-\|B\|,\|B\|]$. Then $\operatorname{dist}(E, \sigma(A))=\|B\|+\delta$ for some $\delta>0$, and $\left\|(A-E)^{-1}\right\|=1 /(\|B\|+\delta)$. In particular, the series

$$
\sum_{n=0}^{\infty}\left((E-A)^{-1} B\right)^{n}
$$

converges to a bounded operator $R$. Let $C=R(A-E)^{-1}$. One easily shows that

$$
C(A+B-E)=(A+B-E) C=I
$$

so $E \notin \sigma(A+B)$. $\square$.

Lemma 5.4 Let $A$ and $B$ be bounded selfadjoint operators such that $\sigma(A) \cap(a, b)=\emptyset$. Then $E \in \sigma(B) \cap(a, b)$ if and only if $1 \in \sigma\left((A-B)(A-E)^{-1}\right)$.

Proof: The lemma follows from the identity

$$
I-(A-B)(A-E)^{-1}=(B-E)(A-E)^{-1},
$$

which holds for $E \in(a, b)$.

We now proceed with the proof.

Let $d(\epsilon, \delta)=\min \{\epsilon / 2, \delta / 2\}$, and let $n_{\epsilon, \delta}$ be a positive integer such that

$$
\sum_{|n| \geq n_{\delta, \epsilon}}|j(n)| \leq d(\epsilon, \delta)
$$

Our first requirement on $L_{\epsilon, \delta}$ is

$$
L_{\epsilon, \delta} \geq n_{\epsilon, \delta}
$$


If $L>L_{\epsilon, \delta}$, we decompose $h_{0}-h_{0}^{L}$ into three parts:

$$
\begin{aligned}
H_{1} & =\sum_{\substack{2 p L-L_{\epsilon, \delta}<k \leq 2 p L \\
k^{\prime}>2 p L}} j\left(k-k^{\prime}\right)\left(\delta_{k^{\prime}}, \cdot\right) \delta_{k}+j\left(k^{\prime}-k\right)\left(\delta_{k}, \cdot\right) \delta_{k^{\prime}}, \\
H_{2}= & \sum_{\substack{-2 p L \leq k<-2 p L+L_{\epsilon, \delta} \\
k^{\prime}<-2 p L}} j\left(k-k^{\prime}\right)\left(\delta_{k^{\prime}}, \cdot\right) \delta_{k}+j\left(k^{\prime}-k\right)\left(\delta_{k}, \cdot\right) \delta_{k^{\prime}} \\
& +\sum_{\substack{-2 p L \leq k \leq 2 p L-L_{\epsilon, \delta} \\
k^{\prime}>2 p L}} j\left(k-k^{\prime}\right)\left(\delta_{k^{\prime}}, \cdot\right) \delta_{k}+j\left(k^{\prime}-k\right)\left(\delta_{k}, \cdot\right) \delta_{k^{\prime}} \\
& +\sum_{\substack{-2 p L+L_{\epsilon, \delta} \leq k \leq 2 p L \\
k^{\prime}<-2 p L}} j\left(k-k^{\prime}\right)\left(\delta_{k^{\prime}}, \cdot\right) \delta_{k}+j\left(k^{\prime}-k\right)\left(\delta_{k}, \cdot\right) \delta_{k^{\prime}}
\end{aligned}
$$

The operators $H_{i}$ are selfadjoint and $H_{1} H_{2}=H_{2} H_{1}=0$. It follows from Lemma 5.2 that $\left\|H_{3}\right\| \leq d(\epsilon, \delta)$. We denote

$$
T_{i}(E) \equiv H_{i}(h-E)^{-1}
$$

It follows from Lemma 5.3 that $\sigma\left(h^{L}\right) \subset \sigma\left(h^{L}+H_{3}\right)+[-d(\epsilon, \delta), d(\epsilon, \delta)]$, Thus, if $E \in S_{L} \cap$ $(a+\epsilon, b-\epsilon)$, then

$$
\mathcal{A}(E) \equiv \sigma\left(h^{L}+H_{3}\right) \cap[E-d(\epsilon, \delta), E+d(\epsilon, \delta)] \neq \emptyset \text {. }
$$

If $\tilde{E} \in \mathcal{A}(E)$, applying Lemma 5.4 with $A=h$ and $B=h^{L}+H_{3}$ and using that $A-B=H_{1}+H_{2}$, we get that

$$
1 \in \sigma\left(T_{1}(\tilde{E})+T_{2}(\tilde{E})\right) .
$$

Note that if $E \in[a+\epsilon / 2, b-\epsilon / 2]$,

$$
\begin{aligned}
\left\|T_{1}(E) T_{2}(E)\right\|+\left\|T_{2}(E) T_{1}(E)\right\| & \leq \frac{2}{\epsilon}\left(\left\|H_{1}(h-E)^{-1} H_{2}\right\|+\left\|H_{2}(h-E)^{-1} H_{1}\right\|\right) \\
& \leq \frac{16}{\epsilon}\left(\sum_{k<0, k^{\prime}>0}\left|j\left(k-k^{\prime}\right)\right|\right)^{2} \sup _{\left|k-k^{\prime}\right|>L_{\epsilon, \delta}}\left|\left(\delta_{k},(h-E)^{-1} \delta_{k^{\prime}}\right)\right| .
\end{aligned}
$$

Since $\gamma>2$, the series converges, and it follows from Proposition 2.2 that

$$
\sup _{E \in[a+\epsilon / 2, b-\epsilon / 2]}\left(\left\|T_{1}(E) T_{2}(E)\right\|+\left\|T_{2}(E) T_{1}(E)\right\|\right) \leq C_{\epsilon} / L_{\epsilon, \delta}
$$

Note also that for any $E \in(a, b)$,

$$
T_{1}(E)^{*} T_{2}(E)=T_{2}(E)^{*} T_{1}(E)=0
$$

Since $T_{1}(\tilde{E})+T_{2}(\tilde{E})$ is compact (in fact trace class), it follows from (5.50) that there is a vector $\psi,\|\psi\|=1$, such that

$$
T_{1}(\tilde{E}) \psi+T_{2}(\tilde{E}) \psi=\psi
$$


It now follows from (5.52) that

$$
1=\left\|T_{1}(\tilde{E}) \psi\right\|^{2}+\left\|T_{2}(\tilde{E}) \psi\right\|^{2}
$$

We conclude that if (5.50) holds for some $L>L_{\epsilon, \delta}$, then the relation

$$
\left\|T_{i}(\tilde{E}) \psi\right\|^{2} \geq 1 / \sqrt{2}
$$

holds for either $i=1$ or $i=2$. Assume for definitness that $i=1$ and let $\phi=T_{1} \psi /\left\|T_{1} \psi\right\|$. Applying $T_{1}$ to both sides of (5.53) we get $T_{1}(\tilde{E}) \phi-\phi=\chi$, where $\chi=-T_{1} T_{2} \psi /\left\|T_{1} \psi\right\|$. It now follows from (5.51) that $\|\chi\| \leq \sqrt{2} C_{\epsilon} / L_{\epsilon, \delta}$. In this way we conclude that if $E \in S_{L} \cap(a+\epsilon, b-\epsilon)$ and $L>L_{\epsilon, \delta}$, then for some $\tilde{E} \in[E-d(\epsilon, \delta), E+d(\epsilon, \delta)]$ and $i \in\{1,2\}$, either $1 \in \sigma\left(T_{i}(\tilde{E})\right)$ or

$$
\left\|\left(T_{i}(\tilde{E})-I\right)^{-1}\right\| \geq L_{\epsilon, \delta} / \sqrt{2} C_{\epsilon} .
$$

We will use this relation shortly.

In the sequel $i=1,2$. Since $H_{i}$ 's are selfadjoint trace class operators and $0 \notin(a, b)$, the spectrum of the operators $h-H_{i}$ within $(a+\epsilon / 4, b-\epsilon / 4)$ consists of finitely many eigenvalues of finite multiplicity. We collect these eigenvalues into the set $\left\{r_{1}, r_{2}, \ldots, r_{k_{\epsilon, \delta}}\right\}$. These eigenvalues do not depend on the choice of $L$. This follows from the observation that for different values of $L$, the operators $h-H_{i}$ are unitarily equivalent. Let us now show that $\sup _{\delta>0} k_{\epsilon, \delta} \leq k_{\epsilon}<\infty$. Indeed, if $r \in(a+\epsilon / 4, b-\epsilon / 4)$ is an eigenvalue of $h-H_{i}$ with normalized eigenvector $\psi$, then

$$
\left(\psi, H_{i}^{2} \psi\right)=\left(\psi,(h-r)^{2} \psi\right) \geq(\epsilon / 4)^{2} .
$$

Therefore,

$$
\operatorname{Tr}\left(H_{1}^{2}\right)+\operatorname{Tr}\left(H_{2}^{2}\right)>2 k_{\epsilon, \delta}(\epsilon / 4)^{2}
$$

On the other hand,

$$
\operatorname{Tr}\left(H_{1}^{2}\right)+\operatorname{Tr}\left(H_{2}^{2}\right) \leq 4 j_{0} \sum_{k \geq 0, k^{\prime}<0}\left|j\left(k-k^{\prime}\right)\right| .
$$

Since $\gamma>2$, the series on the right hand converges, and the statement follows.

Assume now that $E \in S_{L} \cap(a+\epsilon, b-\epsilon)$, and that

$$
E \notin \cup_{l=1}^{k_{\epsilon, \delta}}\left[r_{l}-\delta, r_{l}+\delta\right]
$$

Then for any $\tilde{E} \in \mathcal{A}(E)$, we have that $\tilde{E} \notin \cup_{l=1}^{k_{\epsilon, \delta}}\left[r_{l}-\delta / 2, r_{l}+\delta / 2\right]$ and that (5.50) holds. In particular, since $\operatorname{dist}\left\{\tilde{E}, \sigma\left(h-H_{i}\right)\right\} \geq \min \{\epsilon / 4, \delta / 2\}$,

$$
\left\|\left(h-H_{i}-\tilde{E}\right)^{-1}\right\| \leq 1 / \min \{\epsilon / 4, \delta / 2\} .
$$

Since

$$
T_{i}(\tilde{E})-I=\left(H_{i}-h-\tilde{E}\right)(h-\tilde{E})^{-1},
$$


we have

$$
\left\|\left(T_{i}(\tilde{E})-I\right)^{-1}\right\| \leq\|h-\tilde{E}\|\left\|\left(h-H_{i}-\tilde{E}\right)^{-1}\right\| \leq C / \min \{\epsilon, \delta\} .
$$

where $C$ is a uniform constant. Our last requirement on $L_{\epsilon, \delta}$ is that if $C_{\epsilon}$ is as in (5.51), then

$$
\frac{L_{\epsilon, \delta}}{\sqrt{2} C_{\epsilon}}>C / \min \{\epsilon, \delta\}
$$

This yields that if $L>L_{\epsilon, \delta}$

$$
\left\|\left(T_{i}(\tilde{E})-I\right)^{-1}\right\|<L_{\epsilon, \delta} / \sqrt{2} C_{\epsilon} .
$$

We conclude that if $L_{\epsilon, \delta}$ satisfies (5.49) and (5.56), $L>L_{\epsilon, \delta}, E \in \mathcal{S}_{L} \cap(a+\epsilon, b-\epsilon)$ and (5.55) holds, then for either $i=1$ or $i=2$, Relations (5.54) and (5.57) hold simultaneously for some $\tilde{E} \in \mathcal{A}(E)$, which is imposible. Thus, we must have that $E \in \cup_{l=1}^{k_{\epsilon, \delta}}\left[r_{l}-\delta, r_{l}+\delta\right]$, and the proposition is proven.

\section{Proofs of Theorems 1.1 and 1.2}

We start with the proof of the Theorem 1.2, part 2. We first show that $\Sigma_{c} \cap\left[-\eta_{0}, \eta_{0}\right]=\emptyset$. Let $(\Omega, \mathcal{F}, P)$ be the probability space associated to the model (1.1) (see, e.g., [CFKS] or [CL]). Let $\varepsilon>0$ and $\mu_{\varepsilon}=\eta_{0}-\varepsilon$. Note that

$$
P\left(\left\{\left|v_{\omega}(n)\right|>\mu_{\varepsilon}+j_{0}+\frac{\varepsilon}{2}\right\}\right)=\delta_{\varepsilon}>0 .
$$

Let $a$ be an integer such that $a \delta_{\varepsilon}^{2}>1$. Let us consider the sub-intervals of $\left[a^{n}, a^{n+1}\right],\left[-a^{n+1},-a^{n}\right]$ of the following form:

$$
I_{n}^{(k)}=\left[a^{n}+2(k-1) n+1, a^{n}+2(k-1 / 2) n+1\right], \quad I_{-n}^{(k)}=-I_{n}^{(k)},
$$

where $1 \leq k \leq\left[a\left(a^{n}-1\right) / 2 n\right]-1([\cdot]$ is the greatest integer part). Clearly, these are mutually disjoint intervals of length $n$. Let

$$
A_{n, k}=\left\{\omega:\left|v_{\omega}(i)\right|>\mu_{\varepsilon}+j_{0}+\frac{\varepsilon}{2} \text { for all } i \in I_{n}{ }^{k} \cup I_{-n}^{k}\right\} .
$$

The probability of this event is $P\left(A_{n, k}\right)=\delta_{\varepsilon}^{2 n}$. Let $B_{n}$ be the event that no $A_{n, k}$ take place, i.e.

$$
B_{n}=\Omega \backslash\left(\cup_{k} A_{n, k}\right) .
$$

Clearly,

$$
P\left(B_{n}\right)=\left(1-\delta_{\varepsilon}^{2 n}\right)^{\left[a\left(a^{n}-1\right) / 2 n\right]-1} .
$$

A simple analysis leads to a (rough) bound

$$
P\left(B_{n}\right)=O\left(2^{-a\left(\delta_{\varepsilon}^{2} a\right)^{2 n} / 2 n}\right) .
$$


Since $a \delta_{\varepsilon}^{2}>1$

$$
\sum_{n} P\left(B_{n}\right)<\infty
$$

and the Borel-Cantelli lemma yields that for typical $\omega$ only finitely many events $B_{n}$ take place. This result, combined with Proposition 2.3 yields that for typical $\omega$ there is $N(\omega)$ such that all the conditions of Theorem 3.1 are satisfied with $\mathcal{I}=\left(-\mu_{\varepsilon}, \mu_{\varepsilon}\right)$. We conclude that for a.e. $\omega \in \Omega$ and a.e. $E \in\left(-\mu_{\varepsilon}, \mu_{\varepsilon}\right)$

$$
\lim _{\zeta \downarrow 0} \sum_{m}\left|\left(\delta_{0},\left(h_{\omega}-E-i \zeta\right)^{-1} \delta_{m}\right)\right|^{2}<\infty .
$$

It now follows from the Simon-Wolff theorem [SW] that $\Sigma_{c} \cap\left(-\mu_{\varepsilon}, \mu_{\varepsilon}\right)=\emptyset$. Since $\varepsilon>0$ is arbitrary, the statement follows.

We now show that $\Sigma_{c} \cap\left(\mathbf{R} \backslash \sigma\left(h_{0}+a_{0}\right)\right)=\emptyset$ if $a_{0} \in \operatorname{int}(\mathcal{V})$. Without loss of generality we can assume that $a_{0}=0$. Let $\theta>0$ be such that $(-\theta, \theta) \subset \mathcal{V}$. Let $E_{0} \in \mathbf{R} \backslash \sigma\left(h_{0}\right)$ be a fixed point. Choose $a$ and $b$ such that $E_{0} \in(a, b) \subset \mathbf{R} \backslash \sigma\left(h_{0}\right)$. We will use Proposition 5.1 with $v \equiv 0$ and $p=1$ : For any $\epsilon>0$ and $\delta>0$ we can find $L_{\epsilon, \delta}$ such that

$$
\sigma\left(h_{0}^{L}\right) \cap(a+\epsilon, b-\epsilon) \subset \cup_{l=1}^{k_{\epsilon, \delta}}\left[r_{l}-\delta, r_{l}+\delta\right] .
$$

Since $\sup _{\delta>0} k_{\epsilon, \delta}<k_{\epsilon}<\infty$, we can choose $\epsilon, \delta$, and $x_{0} \in(-\theta / 2, \theta / 2)$ so that

$$
x_{0}+E_{0} \in(a+\epsilon, b-\epsilon) \quad \text { and } \quad x_{0}+E_{0} \notin \cup_{l=1}^{k_{\epsilon, \delta}}\left[r_{l}-\delta, r_{l}+\delta\right]
$$

Clearly, one can take a small open set $\mathcal{I}$ around $E_{0}$ such that for $E \in \mathcal{I}$ Relations (6.60) hold and that

$$
\alpha=\operatorname{dist}\left\{x_{0}+\mathcal{I}, \cup_{l=1}^{k_{\epsilon, \delta}}\left[r_{l}-\delta, r_{l}+\delta\right]\right\}>0 .
$$

Let $\varepsilon=\min \{\alpha / 2, \epsilon / 2\}$. If $L>L_{\epsilon, \delta}$ then

$$
\operatorname{dist}\left(\sigma\left(h_{0}^{L}-x_{0}\right), \mathcal{I}\right\} \geq 2 \varepsilon \text {. }
$$

Note also that

$$
P\left(\left\{\left|v_{\omega}(n)+x_{0}\right|<\varepsilon\right\}\right)=\delta_{\varepsilon}>0 .
$$

We now repeat the probabilistic argument form the begining of this section. Pick an integer $a$ such that $a \delta_{\varepsilon}^{2}>1$. Then for a.e. $\omega$ there exist $N=N(\omega)$ such that, $\forall n>0$, the intervals $\pm\left[a^{N+n}+1, a^{N+n+1}-1\right]$ contain sub-intervals $I_{ \pm n}(\omega)$ of the length $l_{ \pm n}=n$ so that for $k \in I_{ \pm n}(\omega)$, $\left|v_{\omega}(k)+x_{0}\right|<\varepsilon$. By increasing $N(\omega)$, we can assume that $l_{ \pm n}>L_{\epsilon, \delta}$. It then follows from (6.61) and the translation invariance that $\sigma\left(h_{\omega, I_{ \pm n}(\omega)}\right) \cap \mathcal{I}=\emptyset$. Therefore, for a.e. $\omega$, the conditions of Theorem 3.1 are satisfied, and we conclude that for a.e. $\omega$ and a.e. $E \in \mathcal{I}$ Relation (6.59) holds. Thus, $\Sigma_{c} \cap \mathcal{I}=\emptyset$. Since $E_{0} \in \mathbf{R} \backslash \sigma\left(h_{0}\right)$ was arbitrary, the statement follows. 
It should be now obvious how to modify the above argument to show that for if $\gamma>4$ then $\Sigma_{a c}=\emptyset$. For example, let us show that $\Sigma_{a c} \cap\left(\mathbf{R} \backslash \sigma\left(h_{0}\right)\right)=\emptyset$. Choose a sequence $\varepsilon_{k} \downarrow 0$ and note that

$$
P\left(\left\{\left|v_{\omega}(n)\right|<\varepsilon_{k}\right\}\right)=\delta_{\varepsilon_{k}}>0 .
$$

It follows from the above probabilistic argument that for a.e. $\omega$ there exists a sequence of intervals $I_{ \pm k}(\omega)$ which satisfy the condition of Simon-Spencer theorem (Theorem 1.3 of Introduction) and that $\max _{n \in I_{ \pm k}(\omega)}\left|v_{\omega}(n)\right| \leq \varepsilon_{k}$. Thus $\Sigma_{a c} \subset \sigma\left(h_{0}\right)$.

We now turn to the Theorem 1.1, part 2. We again assume that $0 \in \operatorname{int}(\mathcal{V})$. Clearly, we have only to show that $\Sigma_{c} \cap \sigma\left(h_{0}\right)=\emptyset$, since it follows from Theorem 1.2 that $\Sigma_{c} \cap\left(\mathbf{R} \backslash \sigma\left(h_{0}\right)\right)=\emptyset$. Let $\theta>0$ be such that $(-\theta, \theta)$ is contained in $\mathcal{V}$. Let $E_{0} \in\left(\theta_{1}, \theta_{2}\right) \subset(\min \hat{j}(\varphi), \max \hat{j}(\varphi))$ be a given point. We will again show that there exists an interval $\mathcal{I} \ni E_{0}$ such that for a.e. $\omega$ the conditions of Theorem 3.1 are satisfied. Choose $\varepsilon_{0}$ and $p_{0}$ such that Theorem 4.1 holds. Then, since the set points $\left\{\hat{j}(k \pi / p): p>p_{0}, 0 \leq k \leq p\right\}$ is dense in $\sigma\left(h_{0}\right)$, we can find $p>p_{0}$ and $k$ such that $\left|\hat{j}(k \pi / p)-E_{0}\right|<\theta / 4$ and that $k \pi / p \in\left(\theta_{1}, \theta_{2}\right)$. Choose now $\varepsilon$ such that $\varepsilon<\min \left\{\varepsilon_{0}, \theta / 4\right\}$, and let $v_{\varepsilon, p}$ be the periodic potential (4.36). We now use Proposition 5.1: For any $\epsilon>0$ and $\delta>0$ we can find $L_{\epsilon, \delta}$ such that for $L>L_{\epsilon, \delta}$ the spectrum of the operator $h_{0}+v_{\varepsilon, p}$ restricted to $[-2 p L, 2 p L]$ with Dirichlet boundary condition satisfies

$$
\mathcal{S}_{L} \cap(a+\epsilon, b-\epsilon) \subset \cup_{l=1}^{k_{\epsilon, \delta}}\left[r_{l}-\delta, r_{l}+\delta\right],
$$

where $a=\hat{j}(k \pi / p), b=\hat{j}(k \pi / p)+\delta_{\epsilon, k, p}$. Choose now $\epsilon, \delta$ and $x_{0} \in(-\theta / 4, \theta / 4)$ so that (6.60) holds. Clearly, one can take a small open set $\mathcal{I}$ around $E_{0}$ such that for all $E \in \mathcal{I}(6.60)$ holds and that

$$
\operatorname{dist}\left\{\sigma\left(h_{\varepsilon, p}^{L}-x_{0}\right), \mathcal{I}\right\}=\alpha>0 .
$$

Since $x_{0}, x_{0}-\varepsilon \in(-\theta / 2, \theta / 2)$, for each $n$,

$$
P\left(\left\{\left|v_{\omega}(n)+x_{0}-v_{\varepsilon, p}(n)\right|<\frac{\alpha}{2}\right\}\right)>\delta_{\alpha}>0,
$$

where $\delta_{\alpha}$ does not depend on $n$. We now repeat the previous probabilistic arguments to show that the conditions of Theorem 3.1 are satisfied for a.e. $\omega$, and that $\Sigma_{c} \cap \mathcal{I}=\emptyset$. We remark that now the integer $n$ in (6.58) should be replaced by $4 n p$. Since $E_{0} \in(\min \hat{j}(\varphi), \max \hat{j}(\varphi))$ is an arbitrary point, the statement follows.

We leave it as an exercise to the reader to combine the above arguments with Theorem 1.3 of the Introduction to finish the proof of Theorem 1.1, part 1. 


\section{References}

[A] Aizenman M., Localization at Weak Disorder: Some Elementary Bounds. Rev. Math. Phys., 6, 1163 (1994)

[AM] Aizenman M., Molchanov S., Localization at Large Disorder and at Extreme Energies: An Elementary Derivation. Commun. Math. Phys. 157, 245 (1993)

[CL] Carmona R., Lacroix J., Spectral Theory of Random Schrödinger Operators. Birkhauser, Boston 1990.

[CFKS] Cycon H., Froese R., Kirsch W., Simon B., Schrödinger Operators. Springer-Verlag, Berlin-Heidelberg 1987.

[G] Grinshpun V., Localization for Random Potentials Supported on a Subspace. Lett. Math. Phys., 34, 103 (1995)

[GJMS] Gordon Y., Jakšić V., Molchanov S., Simon B., Spectral Properties of Random Schrödinger Operators with Unbounded Potentials. Commun. Math. Phys. 157, 23 (1993)

[JL] Jakšić V., Last Y., in preparation.

[JMP] Jakšić V., Molchanov S., Pastur L., On the Propagation Properties of Surface Waves. Wave Propagation in Complex Media, IMA Vol. Math. Appl, 96, 143 (1998)

[JM] Jakšić V., Molchanov S., On the Surface Spectrum in Dimension Two, preprint.

[K] Katznelson Y., An Introduction to Harmonic Analysis. Dover Publications, New York, 1968.

[Ka] Kato T., Perturbation Theory for Linear Operators. Springer-Verlag, Berlin-Heidelberg 1980.

[KKS] Kirsch W., Kotani S., Simon B., Absence of Absolutely Continuous Spectra for OneDimensional Random but Deterministic Schrödinger Operators. Ann. Inst. H. Poincareé 42, 383 (1985).

[KMP] Kirch W., Molchanov S., Pastur L., One-dimensional Schrödinger Operator with Unbounded Potential. Func. Anal. Prilozhen., 24, 14 (1990)

[M] Molchanov S., Lectures given at Caltech, Spring 1990. 
[M1] Molchanov S., Lectures on Random Media. In Lectures on Probability, ed. P. Bernard, Lecture Notes in Mathematics, 1581, Springer-Verlag, Heidelberg 1994

[S] Simon B., Spectral Analysis of Rank One Perturbations and Applications. CRM Proc. Lecture Notes, 8, AMS, Providence, RI, 1995.

[SS] Simon B., Spencer T., Trace Class Perturbations and the Absence of Absolutely Continuous Spectrum. Commun. Math. Phys. 125, 113 (1989)

[SW] Simon B., Wolff T., Singular Continuous Spectrum Under Rank One Perturbations and Localization for Random Hamiltonians. Commun. Pure Appl. Math. 39, 75 (1986) 